\title{
Stochastic rotation dynamics. II. Transport coefficients, numerics, and long-time tails
}

\author{
T. Ihle ${ }^{1,2, *}$ and D. M. Kroll ${ }^{1}$ \\ ${ }^{1}$ Supercomputing Institute, University of Minnesota, 599 Walter Library, 117 Pleasant Street S.E., Minneapolis, Minnesota 55455, USA \\ ${ }^{2}$ IFF, Theorie II, Forschungszentrum Jülich, 52425 Jülich, Germany
}

(Received 27 November 2002; published 12 June 2003)

\begin{abstract}
A discrete-time projection operation technique was used to derive the Green-Kubo relations for the transport coefficients of a recently introduced stochastic model for fluid dynamics in a previous paper (Part 1). The most important feature of the analysis was the incorporation of a new grid shifting procedure which was shown to guarantee Galilean invariance for arbitrary Mach number and temperature. This paper (Part 2) contains a detailed analysis of the transport coefficients of this model. An exact calculation of the first terms in the stress correlation function in the limit of infinite particle density is presented, which explicitly accounts for the cell structure introduced to define the collision environment. It is also shown that this cell structure can lead to additional contributions to the transport coefficients even at large mean free paths. Explicit expressions for all transport coefficients are derived and compared with simulation results. Long-time tails in the velocity, stress, and heat-flux autocorrelation functions are measured and shown to be in excellent agreement with the predictions of mode-coupling theory.
\end{abstract}

DOI: 10.1103/PhysRevE.67.066706

PACS number(s): 02.70.Ns, 47.11.+j, 05.40.-a

\section{INTRODUCTION}

In a previous paper, Part 1 of this series, a discrete-time projection operator technique was used to derive the GreenKubo relations for the transport coefficients of a recently introduced stochastic model for fluid dynamics with continuous velocities and efficient multiparticle collisions. It was also shown how random shifts of the collision environment could be used to ensure Galilean invariance for arbitrary Mach number and temperature. In this paper (Part 2), we present a detailed analytical and numerical analysis of the Green-Kubo relations derived in Part 1. Several approximations will be discussed in detail and compared with simulation results. The analytical calculations assume that the equal time correlations are those of an ideal gas, but no assumptions are made regarding molecular chaos; the correlations which can develop at small mean free path are explicitly accounted for. The only other approximation we make is to neglect fluctuations in the number of particles in a cell. This amounts to neglecting terms of the order of $e^{-M}$, where $M$ is the average number of particles in a cell, and is therefore justified in all practical calculations, where $M \geqslant 5$.

Section II of this paper contains an analysis of the kinetic contributions to the shear viscosity. An explicit calculation of the kinematic shear viscosity valid in the continuum limit, $\lambda / a \rightarrow \infty$, is presented in Sec. II A, and finite cell size (or finite $\lambda / a$ ) corrections are discussed in subsequent sections. In particular, it is shown in Sec. II B 4 that there are corrections proportional to $(a / \lambda)^{2}$ to the shear viscosity which resolve previous discrepancies between theory and simulation for $\alpha \approx 90^{\circ}$ in two dimensions. An analysis of the rotational (stochastic collision) and mixed contributions to the shear viscosity is presented in Sec. III. A simple approximate expression for the rotational contribution to the viscosity valid

\footnotetext{
*Present address: Institut für Computeranwendungen 1, Universität Stuttgart, Pfaffenwaldring 27, 70569 Stuttgart, Germany.
}

in the limit of small mean free path is presented in Sec. III A, while subsequent sections discuss the equal time contributions to the rotational and mixed stress correlations and present a comparison with simulation results. In Sec. IV, it is shown that the bulk viscosity is zero for this model, and the thermal diffusivity and self-diffusion constant are discussed in Secs. V and VI, respectively. Results for the long-time tails in the stress correlation functions and corresponding transport coefficients are presented in Sec. VII and shown to be in excellent agreement with the predictions of modecoupling theory. The work is summarized in Sec. VIII.

\section{ANALYSIS OF THE KINETIC CONTRIBUTIONS TO THE SHEAR VISCOSITY}

\section{A. Continuum approximation, $\lambda / a \rightarrow \infty$}

The Green-Kubo relation for the viscosities is given in Eq. (56) of Part 1 of this series, and the relevant reduced fluxes are defined in Eq. (55) of that paper. Restricting ourselves to two dimensions, the shear viscosity is obtained if we take $\hat{\mathbf{k}}$ in the $y$ direction and $\alpha=\beta=1$, so that

$$
\nu=\frac{\tau}{N k_{B} T} \sum_{t=0}^{\infty}{ }^{\prime}\left\langle I_{2}(\hat{y}, 0) \mid I_{2}(\hat{y}, t)\right\rangle .
$$

For large mean free path, one expects contributions from the term proportional to $\hat{\mathbf{k}} \cdot \Delta \boldsymbol{\xi}_{j}^{s}$ in Eq. (55) of Part 1 to be negligible. Furthermore, for $\lambda / a \rightarrow \infty$, we assume that $\Delta \xi_{j x}$ can be replaced by $\tau v_{j x}$ in the ensemble average; the validity of these approximations is discussed in Sec. II B of this paper. We therefore need to evaluate $C_{n} \equiv\left\langle I_{2}(\hat{y}, 0) \mid I_{2}(\hat{y}, n \tau)\right\rangle$, where $I_{2}(\hat{y}, t)=\sum_{i=1}^{N} v_{i x} v_{i y}$. The evolution of the velocity of particle $i$ during a time step is described by

$$
v_{i x}(t+\tau)=u_{\xi x}(t)+c\left[v_{i x}(t)-u_{\xi x}(t)\right]+s\left[v_{i y}(t)-u_{\xi y}(t)\right]
$$


and

$$
v_{i y}(t+\tau)=u_{\xi y}(t)+c\left[v_{i y}(t)-u_{\xi y}(t)\right]-s\left[v_{i x}(t)-u_{\xi x}(t)\right],
$$

with $c=\cos (\alpha), s=\sin (\alpha)$, and $\mathbf{u}_{\xi}=(1 / M) \sum_{k \in \xi} \mathbf{v}_{k}$, where the sum runs over all particles in the cell occupied by particle $i$ at $t=n \tau$. Consider first

$$
\begin{aligned}
C_{1}= & \sum_{i j}\left\langle v_{i x} v_{i y}\left[u_{\xi x}+c\left(v_{j x}-u_{\xi x}\right)+s\left(v_{j y}-u_{\xi y}\right)\right]\right. \\
& \left.\times\left[u_{\xi y}+c\left(v_{j y}-u_{\xi y}\right)-s\left(v_{j x}-u_{\xi x}\right)\right]\right\rangle,
\end{aligned}
$$

where $\quad \mathbf{v}_{i} \equiv \mathbf{v}_{i}(0) \quad$ and $\quad \mathbf{u}_{\xi} \equiv \mathbf{u}_{\xi}(0)$. Using $\left\langle v_{i \alpha} v_{j \beta}\right\rangle$ $=\delta_{i j} \delta_{\alpha \beta} k_{B} T$ and $\langle s\rangle=0$, one finds that there are both diagonal and off-diagonal contributions to $C_{1}$. The off-diagonal contribution comes from particles $j$ which are in the same cell as particle $i$ at $t=0$. In this case, $\mathbf{v}_{j}(\tau)$ has a "projection" on $\mathbf{v}_{i}(0)$ through the mean cell velocity. One finds

$$
\begin{aligned}
\left\langle v_{i x} v_{i y} v_{j x}(\tau) v_{j y}(\tau)\right\rangle= & \left\langlev _ { i x } v _ { i y } [ ( 1 - c ) v _ { i x } - s v _ { i y } ] \left[(1-c) v_{i y}\right.\right. \\
& \left.\left.+s v_{i x}\right]\right\rangle / M^{2}=2\left(\frac{k_{B} T}{M}\right)^{2} c(c-1) .
\end{aligned}
$$

The diagonal contribution is

$$
\begin{aligned}
\left\langle v_{i x} v_{i y} v_{i x}(\tau) v_{i y}(\tau)\right\rangle= & \left\langle v_{i x} v_{i y}\left[\zeta_{1} v_{i x}+\zeta_{2} v_{i y}\right]\right. \\
& \left.\times\left[\zeta_{1} v_{i y}-\zeta_{2} v_{i x}\right]\right\rangle,
\end{aligned}
$$

where $\zeta_{1}=1 / M+c(1-1 / M)$ and $\zeta_{2}=s(1-1 / M)$. Performing the averages, one has

$$
\left\langle v_{i x} v_{i y} v_{i x}(\tau) v_{i y}(\tau)\right\rangle=\left(k_{B} T\right)^{2} \zeta,
$$

where

$$
\zeta=\zeta_{1}^{2}-\zeta_{2}^{2}=\left[\frac{1}{M}+c\left(1-\frac{1}{M}\right)\right]^{2}-s^{2}\left(1-\frac{1}{M}\right)^{2} .
$$

Since there are $M-1$ off-diagonal contributions, one finds

$$
C_{1}=N\left(k_{B} T\right)^{2}[\zeta+\eta],
$$

where $\eta=2(M-1) c(c-1) / M^{2}$. Note that the leading diagonal contribution is $O(1)$, while that of the off-diagonal contribution is $O(1 / M)$.

The behavior over longer-time intervals can be analyzed in a similar fashion. Consider $C_{2}$. Following the arguments of the last paragraph, there is a diagonal contribution proportional to $\zeta^{2}$ and an off-diagonal contribution proportional to $2 \eta \zeta$, since at each time step, $M-1$ particles become correlated with particle $i$, and particle $j$ can become correlated with particle $i$ at either of the two time steps. Note, however, that there are now additional-higher order-contributions which arise, for example, when particle $j$ becomes correlated with particle $k$ which then becomes correlated with particle $i$. It is easy to see that these contributions carry additional factors of $1 / M$ and are thus of higher order than the diagonal and direct off-diagonal contributions considered above. However, these higher off-diagonal contributions can be summed in the geometric series

$$
C_{n} / N\left(k_{B} T\right)^{2}=[\zeta+\eta]^{n} \approx \zeta^{n}+n \eta \zeta^{n-1}+\cdots,
$$

so that

$$
\begin{aligned}
\nu & =k_{B} T \tau\left(\frac{1}{2}+\sum_{j=1}^{\infty}[\zeta+\eta]^{j}\right) \\
& =\frac{k_{B} T \tau}{2}\left(\frac{1}{(1-1 / M) \sin ^{2}(\alpha)}-1\right) .
\end{aligned}
$$

For $\alpha=90^{\circ}$, this result agrees, apart from exponentially small $O\left(e^{-M}\right)$ corrections arising from fluctuations in the number of particles in a cell, with the expression given by Malevanets and Kapral [1,2]. We have shown previously [3] that the viscosity measured in simulations is much larger than this value for $\alpha \simeq 90^{\circ}$. This discrepancy is resolved in the following sections.

\section{B. Lattice effects, $\lambda / a$ finite}

In this section we go beyond the continuum approximation discussed in Sec. II A and consider explicitly the effect of the cell structure used to determine the collision neighbors. This is particularly important for small mean free path, where neither the decay rate of the kinetic stress correlations nor its dependence on the rotation angle is correctly described by the continuum approximation. There are also conditions (large $M, \alpha \approx 90^{\circ}$ ) for which finite cell size corrections are important, even for large mean free path. It is shown how accurate approximations for the transport coefficients can be obtained in these cases even when the assumption of molecular chaos is not valid and where the details of the lattice structure are important. The resulting expressions are shown to be in good agreement with simulation data, and therefore clarify the source of the shortcomings of the approximations discussed in the Sec. II A.

In order to go beyond the continuum approximation when evaluating the Green-Kubo relation (1) for the shear viscosity, we need to consider the full reduced flux $I_{2}(\hat{y}, t)$ given in Eq. (54) of Part 1 of this series. Explicitly,

$$
I_{2}(\hat{y}, t)=-\frac{1}{\tau} \sum_{j}\left[v_{j x}(t) \Delta \xi_{j x}(t)+\Delta v_{j x}(t) \Delta \xi_{j x}^{s}(t)\right] .
$$

Correlation functions involving $\Delta \xi$ and $\Delta \xi^{s}$ therefore need to be evaluated. In the following sections we consider these terms, starting with equal time correlations.

\section{Calculation of $\left\langle\Delta \xi_{i \beta} v_{i \varepsilon}^{m}\right\rangle$}

Assume, as in an ideal gas, that the probability distributions for particle coordinates and velocities are decoupled, and that the particles are homogeneously distributed in space. We proceed by first averaging over all particle positions at fixed velocities. For this purpose, all possible propa- 
gation distances $v_{i \beta} \tau$ of a particle (in $\beta$ direction) are divided into intervals of cell size $a$. Averaging over the particle's spatial coordinate for fixed velocity $v_{i \beta}$, the probability of a cell displacement $\Delta \xi_{i \beta}=n a$ is

$$
p_{n}=\left[v_{i \beta} \tau-a(n-1)\right] / a
$$

if the velocity is in the interval $(n-1) a \leqslant v_{i \beta} \tau<n a$. The probability of a cell displacement $\Delta \xi_{i \beta}=(n-1) a$ is $q_{n}=1$ $-p_{n}$. It is easy to see that $\left\langle\Delta \xi_{i \beta} v_{i \varepsilon}^{m}\right\rangle=0$ for $\beta \neq \varepsilon$. For $\beta$ $=\varepsilon$,

$$
\begin{aligned}
\left\langle\Delta \xi_{i \beta} v_{i \beta}^{m}\right\rangle= & a \sum_{n=-\infty}^{\infty} \int_{(n-1) a / \tau}^{n a / \tau} w\left(v_{i \beta}\right) v_{i \beta}^{m} \\
& \times\left\{n p_{n}+(n-1) q_{n}\right\} d v_{i \beta},
\end{aligned}
$$

where $w\left(v_{i \alpha}\right)$ is the Boltzmann distribution. Since

$$
a n p_{n}+a(n-1) q_{n}=v_{i \beta} \tau,
$$

Eq. (14) reduces to a single integral, and we have

$$
\left\langle\Delta \xi_{i \beta} v_{i \beta}^{m}\right\rangle=\tau\left\langle v_{i \beta}^{m} v_{i \beta}\right\rangle,
$$

so that $\Delta \xi_{i \beta}$ can be replaced by $\tau v_{i \beta}$ in averages that are linear in $\Delta \xi$. We measured $\left\langle\Delta \xi_{i x} v_{i x}\right\rangle$ in a simulation, and found, in agreement with these results, that it is equal to $\tau k_{B} T$ within statistical error. Averages such as $\left\langle\left(\Delta \xi_{i \beta}\right)^{2}\right\rangle$, on the other hand, which are not linear in $\Delta \xi$, need to be calculated explicitly, since they differ from what would be obtained by substituting $\tau v_{i \beta}$ for $\Delta \xi_{i \beta}$ in the average.

\section{Calculation of $\left\langle\left(\Delta \xi_{i x}\right)^{2}\right\rangle$}

For convenience, we omit the particle index $i$ in this section. We also assume that the particle density is homogeneous and that the static correlation functions are those of an ideal gas, e.g., $\left\langle v_{i \alpha} r_{j \beta}\right\rangle=0$. Consider a particle that moves from position $r_{x}=x_{0}$ to $x_{0}+\tau v_{x}$ in one time step. For fixed $v_{x}$, the probability that $\Delta \xi_{x}=n a$ is

$$
\begin{aligned}
P_{v_{x}}\left(\Delta \xi_{x}=n a\right)= & \frac{1}{a} \int_{0}^{a} d x_{0} \Theta\left(x_{0}+v_{x} \tau-n a\right) \\
& \times\left\{1-\Theta\left(x_{0}+v_{x} \tau-[n+1] a\right)\right\},
\end{aligned}
$$

where we have averaged over the initial position $x_{0}$ of the particle. For $(n-1) a \leqslant v_{x} \tau<n a$, Eq. (17) reduces to $P_{v_{x}}\left(\Delta \xi_{x}=n a\right)=p_{n}$, where $p_{n}$ is given by Eq. (13), as it should.

Using Eq. (17), we have

$$
\begin{aligned}
\left\langle\left(\Delta \xi_{x}\right)^{2}\right\rangle & =\sum_{n=-\infty}^{\infty} \int_{-\infty}^{\infty} d v_{x} P_{v_{x}}\left(\Delta \xi_{x}=n a\right)(n a)^{2} w\left(v_{x}\right) \\
& =\frac{1}{a} \int_{0}^{a} d x_{0} \sum_{n=-\infty}^{\infty} \int_{\left(n a-x_{0}\right) / \tau}^{\left[(n+1) a-x_{0}\right] / \tau}(a n)^{2} w\left(v_{x}\right) d v_{x},
\end{aligned}
$$

where

$$
w\left(v_{x}\right)=\frac{1}{\sqrt{2 \pi k_{B} T}} \exp \left\{-\frac{v_{x}^{2}}{2 k_{B} T}\right\}
$$

is the Boltzmann distribution. The Poisson sum formula [4]

$$
\sum_{n=-\infty}^{\infty} g(n)=\sum_{m=-\infty}^{\infty} \int_{-\infty}^{\infty} g(\phi) e^{-2 \pi i m \phi} d \phi
$$

can now be used to transform Eq. (18) into

$$
\left\langle\left(\Delta \xi_{x}\right)^{2}\right\rangle=a \int_{0}^{a} d x_{0} \sum_{n=-\infty}^{\infty} \int_{-\infty}^{\infty} \phi^{2} h(\phi) e^{-2 \pi i n \phi} d \phi,
$$

with

$$
h(\phi)=\int_{\left(\phi a-x_{0}\right) / \tau}^{\left[(\phi+1) a-x_{0}\right] / \tau} w\left(v_{x}\right) d v_{x} .
$$

A partial integration with respect to $\phi$ is next performed to eliminate the integral in $h(\phi)$. The resulting $n=0$ term in the sum in Eq. (21), $S_{0}$, is

$$
\begin{aligned}
S_{0}= & -\frac{a^{2}}{\tau} \int_{0}^{a} d x_{0} \int_{-\infty}^{\infty} \frac{\phi^{3}}{3}\left\{w\left[\left((\phi+1) a-x_{0}\right) / \tau\right]\right. \\
& \left.-w\left[\left(\phi a-x_{0}\right) / \tau\right]\right\} d \phi .
\end{aligned}
$$

The integrals can be performed to obtain

$$
S_{0}=\frac{a^{2}}{6}+\tau^{2} k_{B} T
$$

Consider now the $n \neq 0$ terms in Eq. (21) and introduce the quantity $\Gamma=2 \pi i n$. Denoting this sum by $S_{P}$, we have

$$
S_{P}=\frac{a^{2}}{\tau} \sum_{n \neq 0} \int_{0}^{a} d x_{0} \int_{-\infty}^{\infty}\left(-\frac{2}{\Gamma^{2}}-\frac{2}{\Gamma} \phi+\frac{1}{\Gamma}\right) e^{-\phi \Gamma} w_{0} d \phi,
$$

where the property $e^{ \pm \Gamma}=1$ was used, and $w_{0}=w([\phi a$ $\left.\left.-x_{0}\right] / \tau\right)$. Using

$$
\int_{0}^{a} d x_{0} \int_{-\infty}^{\infty} e^{-\phi \Gamma} w_{0} d \phi=0
$$

and 


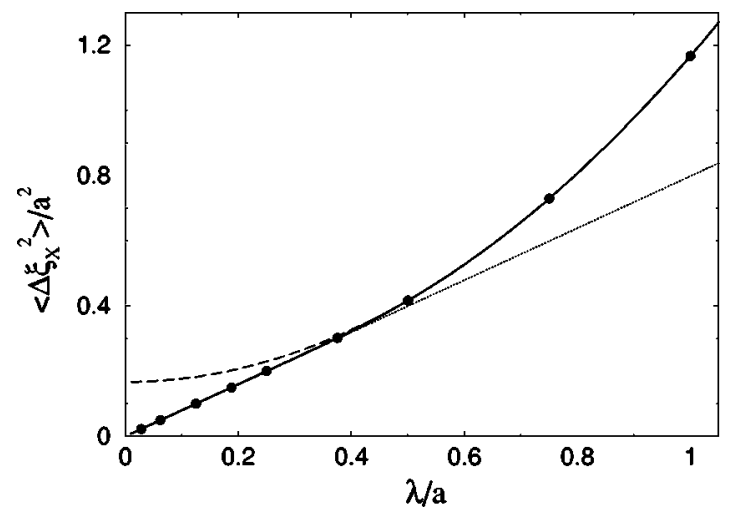

FIG. 1. $\left\langle\Delta \xi_{x}^{2}\right\rangle / a^{2}$ as a function of $\lambda / a$. The bullets are simulation data obtained on a $64 \times 64$ grid; the solid line is expression (28); the dotted lines, approximation (29) for $\lambda / a \ll 1$; and the dashed line is approximation (30) for $\lambda / a \gg 1$.

$$
\int_{0}^{a} d x_{0} \int_{-\infty}^{\infty} \phi e^{-\phi \Gamma} w_{0} d \phi=-\frac{\tau}{2 \pi i n} \exp \left[-2 \pi^{2} n^{2}(\lambda / a)^{2}\right],
$$

one finds the final result

$$
\begin{aligned}
\left\langle\left(\Delta \xi_{i x}\right)^{2}\right\rangle= & S_{0}+S_{P}=a^{2}\left[\frac{1}{6}+\left(\frac{\lambda}{a}\right)^{2}-\frac{1}{\pi^{2}} \sum_{n=1}^{\infty}\right. \\
& \left.\times \frac{1}{n^{2}} \exp \left[-2 \pi^{2} n^{2}(\lambda / a)^{2}\right]\right],
\end{aligned}
$$

where $\lambda=\tau \sqrt{k_{B} T}$ is the mean free path. For small mean free path, $\lambda / a \ll 1$,

$$
\left\langle\left(\Delta \xi_{i x}\right)^{2}\right\rangle \approx a \lambda \sqrt{2 / \pi}
$$

while for large mean free path,

$$
\left\langle\left(\Delta \xi_{i x}\right)^{2}\right\rangle \approx \frac{1}{6} a^{2}+\lambda^{2}
$$

Note that the small term $\sim a^{2} / 6$ in Eq. (30) is absent in the continuum approximation discussed in Sec. II A. We will see later that this correction can, in certain cases, provide the dominant contribution to the viscosity.

These results for $\left\langle\left(\Delta \xi_{i x}\right)^{2}\right\rangle / a^{2}$ are compared with simulation data in Fig. 1. The solid line is expression (28), and the dotted and dashed lines are plots of Eqs. (29) and (30), respectively. As can be seen, the agreement is excellent for all mean free paths. It can also be seen that the large mean free path approximation (30) is valid for $\lambda / a \gtrsim 0.4$, and that for small mean free path, Eq. (29) is accurate for $\lambda / a \leqq 0.4$.

\section{Calculation of $\left\langle\Delta \xi_{i x} \Delta \xi_{i x}^{s}\right\rangle$}

Since $\Delta \xi_{i x}$ does not depend on the random shift, we first determine the average of $\Delta \xi_{i x}^{s}$ over random shifts $\delta$ at fixed particle coordinate and velocity, $\left\langle\left.\Delta \xi_{i x}^{s}\right|_{X}\right\rangle_{\delta}$. For $n a \leqslant X \equiv x_{0}$ $+v_{x} \tau<(n+1) a$,

$$
\begin{aligned}
& \left\langle\Delta \xi_{i x}^{s} \mid X\right\rangle_{\delta} \\
& \quad=\frac{1}{a} \int_{-a / 2}^{a / 2}\{\Theta(n a-X-\delta)-\Theta(X+\delta-[n+1] a)\} d \delta \\
& \quad=\{(n+1 / 2) a-X\} .
\end{aligned}
$$

It follows that

$$
\begin{aligned}
\left\langle\Delta \xi_{i x} \Delta \xi_{i x}^{s}\right\rangle= & \frac{1}{a} \int_{0}^{a} d x_{0} \sum_{n=-\infty}^{\infty} a n \int_{\left(n a-x_{0}\right) / \tau}^{\left[(n+1) a-x_{0}\right] / \tau}\left\{a\left(n+\frac{1}{2}\right)\right. \\
& \left.-\left[x_{0}+v_{x} \tau\right]\right\} w\left(v_{x}\right) d v_{x}
\end{aligned}
$$

As in the preceding section, we use the Poisson sum formula (20) and integrate by parts. The resulting $n=0$ term is

$$
\begin{aligned}
T_{0}= & \frac{a^{2}}{12 \tau} \int_{0}^{a} d x_{0} \int_{-\infty}^{\infty}\left\{\left(2 \phi^{3}+3 \phi^{2}\right) w_{1}\right. \\
& \left.+\left(-2 \phi^{3}+3 \phi^{2}\right) w_{0}\right\} d \phi,
\end{aligned}
$$

with $w_{0}=w\left(\left[\phi a-x_{0}\right] / \tau\right)$ and $w_{1}=w\left(\left[(\phi+1) a-x_{0}\right] / \tau\right)$. Performing the integrals, one finds

$$
T_{0}=\frac{a^{2}}{12} .
$$

The other $n \neq 0$ terms are

$$
T_{P}=\frac{a^{2}}{\tau} \sum_{n \neq 0} \int_{0}^{a} d x_{0} \int_{-\infty}^{\infty}\left(-\frac{2}{\Gamma^{2}}-\frac{\phi}{\Gamma}+\frac{1}{2 \Gamma}\right) e^{-\phi \Gamma} w_{0} d \phi,
$$

where again $\Gamma=2 \pi i n$. Using Eqs. (26) and (27), we find

$$
\left\langle\Delta \xi_{i x} \Delta \xi_{i x}^{s}\right\rangle=T_{0}+T_{P}=\frac{1}{2}\left[\left\langle\left(\Delta \xi_{i x}\right)^{2}\right\rangle-\lambda^{2}\right],
$$

where $\left\langle\left(\Delta \xi_{i x}\right)^{2}\right\rangle$ is given by Eq. (28). In the limit of small mean free path, $\left.\left\langle\Delta \xi_{i x} \Delta \xi_{i x}^{s}\right\rangle \sim a \lambda \sqrt{2 / \pi}\right)$, and for $\lambda / a \rightarrow \infty$, $\left\langle\Delta \xi_{i x} \Delta \xi_{i x}^{s}\right\rangle \sim a^{2} / 12$.

\section{Calculation of $\left\langle\Delta \xi_{i y}^{(0)} v_{i x}^{(0)} \Delta \xi_{i y}^{(1)} v_{i x}^{(1)}\right\rangle$ for $\alpha=90^{\circ}$}

The following calculations are performed in the limit $M$ $\rightarrow \infty$, so that we can use the simplified evolution equations

$$
v_{i x}^{(1)}=c v_{i x}^{(0)}+z s v_{i y}^{(0)}
$$

and

$$
v_{i y}^{(1)}=c v_{i y}^{(0)}-z s v_{i x}^{(0)},
$$

where $c=\cos (\alpha), s=\sin (\alpha)$, and the number $z= \pm 1$ describes the stochastic nature of the rotation. A detailed investigation for finite $M$, and a discussion of the limit $M \rightarrow \infty$, is tedious and will be given elsewhere [5]. For $\alpha=90^{\circ}$, the case considered here, Eqs. (37) and (38) reduce to

$$
v_{i x}^{(1)}=z v_{i y}^{(0)}
$$


and

$$
v_{i y}^{(1)}=-z v_{i x}^{(0)}
$$

Given Eqs. (39) and (40), $C_{1}=\left\langle\Delta \xi_{i y}^{(0)} v_{i x}^{(0)} \Delta \xi_{i y}^{(1)} v_{i x}^{(1)}\right\rangle$ can be written as

$$
\begin{aligned}
C_{1}= & -a \int_{0}^{a} d x_{0} \sum_{n, m=-\infty}^{\infty} \int_{\left(n a-y_{0}\right) / \tau}^{\left[(n+1) a-y_{0}\right] / \tau} d v_{y} \\
& \times \int_{b_{0}}^{b_{1}} d v_{x} n m w\left(v_{x}\right) w\left(v_{y}\right) v_{x} v_{y},
\end{aligned}
$$

where all velocities are at equal time, so that we have dropped the index $(0)$. Note that the average over $z= \pm 1$ has already been performed. The limits on the inner integral are

$$
b_{0}=\left[(m+n) a-y_{0}-v_{y} \tau\right] / \tau
$$

and

$$
b_{1}=\left[(m+n+1) a-y_{0}-v_{y} \tau\right] / \tau .
$$

If the integral over $v_{x}$ is performed, the sum over $m$ can be simplified, and one obtains

$$
\begin{aligned}
C_{1}= & -\frac{a f}{2 r} \int_{0}^{a} d y_{0} \sum_{n, m=-\infty}^{\infty} n \int_{\left(n a-y_{0}\right) / \tau}^{\left[(n+1) a-y_{0}\right] / \tau} d v_{y} v_{y} w\left(v_{y}\right) \\
& \times \exp \left(-r b_{0}^{2}\right)
\end{aligned}
$$

where $r=1 /\left(2 k_{B} T\right)$ and $f=1 / \sqrt{2 \pi k_{B} T}$. Making the transformation $v=v_{y}-n a / \tau+y_{0} / \tau$, the limits of the integral over $v_{y}$ become independent of $y_{0}$, and the integral over $y_{0}$ can be performed, yielding

$$
\begin{aligned}
C_{1}= & \frac{a f^{2} \tau}{4 r^{2}} \sum_{m=-\infty}^{\infty} \int_{0}^{a / \tau} d v \exp \left\{-r[(m a-v \tau) / \tau]^{2}\right\} \\
& \times \sum_{n=-\infty}^{\infty} n\left[\exp \left(-r c_{0}^{2}\right)-\exp \left(-r c_{1}^{2}\right)\right],
\end{aligned}
$$

where $c_{0}=(n a+v \tau) / \tau$ and $c_{1}=[(n-1) a+v \tau] / \tau$. Simplifying the sum over $n$ yields

$$
C_{1}=-\frac{a^{2} k_{B} T}{2 \pi} \int_{0}^{1} d v\left[\sum_{n=-\infty}^{\infty} \exp \left(-\frac{a^{2}}{2 \lambda^{2}}(n+v)^{2}\right)\right]^{2} .
$$

The integral over $v$ can be performed in conjunction with one of the sums to obtain the result

$$
C_{1}=-\frac{k_{B} T a \lambda}{2 \sqrt{\pi}} \sum_{m=-\infty}^{\infty} \exp \left[-\left(\frac{a n}{2 \lambda}\right)^{2}\right] \text {, }
$$

which converges rapidly for small mean free path. The Poisson sum formula, Eq. (20), can be used to obtain the expression

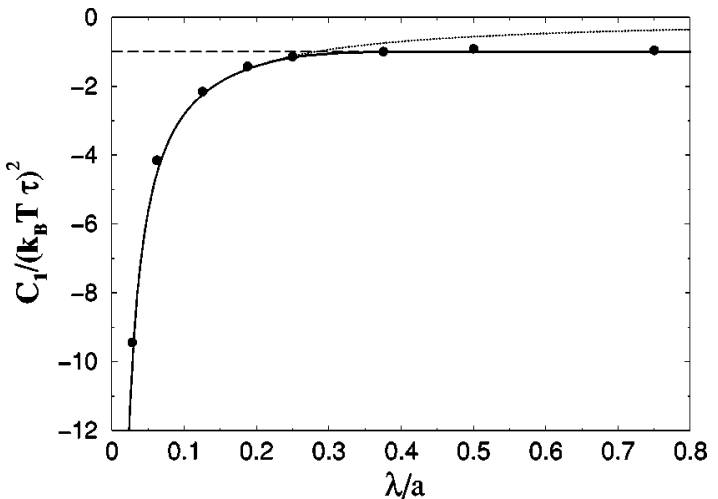

FIG. 2. The dimensionless kinetic stress correlation function $C_{1} /\left(k_{B} T \tau\right)^{2}$, where $C_{1} \equiv\left\langle\Delta \xi_{y}^{(0)} v_{x}^{(0)} \Delta \xi_{y}^{(1)} v_{x}^{(1)}\right\rangle$, as a function of $\lambda / a$. The bullets are simulation data; the solid line is the analytical expression for $\alpha=90^{\circ}$ and $M \rightarrow \infty$, Eq. (47). The dotted line is the small mean free path approximation $C_{1}=-k_{B} \operatorname{Ta} \lambda /(2 \sqrt{\pi})$, and the dashed line is the large mean free path approximation $C_{1}=$ $-\left(k_{B} T \tau\right)^{2}$. Simulation parameters: $M=35, \alpha=90^{\circ}, k_{B} T=4, L$ $=64$.

$$
C_{1}=-\left(k_{B} T \tau\right)^{2} \sum_{m=-\infty}^{\infty} \exp \left[-\left(\frac{2 \pi n \lambda}{a}\right)^{2}\right]
$$

which converges rapidly for large mean free path.

From Eq. (48), it follows that $C_{1} \rightarrow-\left(k_{B} T \tau\right)^{2}$ for $\lambda / a$ $\rightarrow \infty$, which is the result obtained by replacing $\Delta \xi_{y}$ by $\tau v_{y}$ in the definition of $C_{1}$. This result suggests that this might also be true for correlations at larger time, i.e., for $C_{n}, n>1$. Simulations confirm this hypothesis for $\lambda / a \gtrsim 0.4$, see Fig. 9(a).

Figure 2 contains a comparison of the normalized correlation function $C_{1} /\left(\tau k_{B} T\right)^{2}$ with simulation data for $\alpha$ $=90^{\circ}$. The agreement is very good for all mean free paths. The small deviations arise from the fact that the results of this section were derived in the $M \rightarrow \infty$ limit, while $M=35$ in the simulations. Equation (8) suggests that the correction factor is approximately $(1-2 / M)$.

Since $\left\langle\left(\Delta \xi_{x} v_{y}\right)^{2}\right\rangle=\left\langle\Delta \xi_{x}^{2}\right\rangle(\lambda / \tau)^{2}$, the results of this section predict that the initial decay rate of the stress correlations, $g \equiv C_{1} /\left\langle\left(\Delta \xi_{x} v_{y}\right)^{2}\right\rangle$, is given by

$$
g= \begin{cases}-1 /\left[1+a^{2} /\left(6 \lambda^{2}\right)\right] & \text { for } \lambda / a \gg 1 \\ -1 /(2 \sqrt{2}) & \text { for } \lambda / a \ll 1\end{cases}
$$

in the limit $M \rightarrow \infty$. In the continuum approximation, for $\lambda / a \rightarrow \infty$, it follows that $g=-1$, which corresponds to zero viscosity. For finite $\lambda / a$, however, $|g|<1$ and the viscosity is finite. Figure 3(a) shows the predicted dependence of $g$ on the mean free path.

For the large mean free path, the only significant difference between the continuum approximation (discussed in Sec. II A) and the calculation presented here occurs in the $t$ $=0$ contribution, 

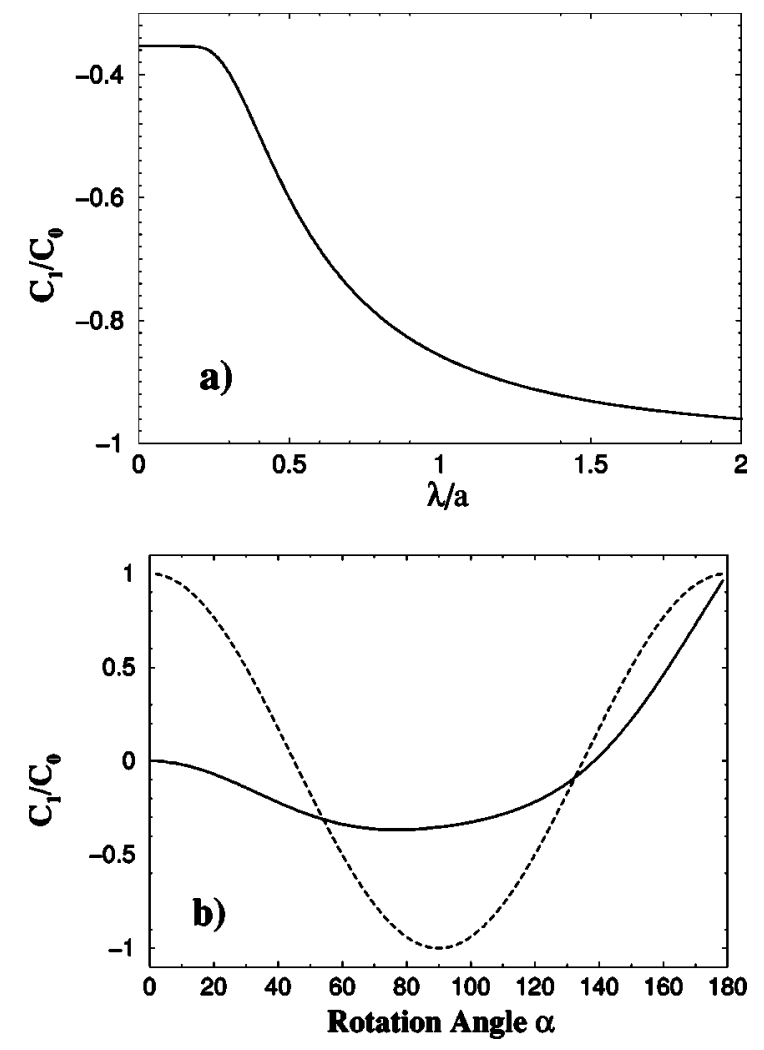

FIG. 3. Plots of analytic expressions for the initial decay rate of the kinetic stress correlations, $g \equiv C_{1} / C_{0}$. (a) $g$ as a function of $\lambda / a$ for $\alpha=90^{\circ}$ and $M \rightarrow \infty$. Note the plateau for $\lambda / a \approx 0.25$ and the subsequent rapid decrease in $g$. (b) $g$ as a function of $\alpha$. The solid line is a plot of Eq. (59), which describes the behavior for $\lambda / a$ $\ll 1$. The dashed curve is the continuum result, $g=\cos ^{2}(\alpha)$, valid for $\lambda / a \rightarrow \infty$ and large $M$. Here and in the following all angles are measured in degrees.

$$
\begin{aligned}
C_{0} & \equiv\left\langle I_{2}(\hat{y}, 0) \mid I_{2}(\hat{y}, 0)\right\rangle \approx \frac{1}{\tau^{2}} \sum_{i j}\left\langle v_{i x} \Delta \xi_{i y} v_{j x} \Delta \xi_{j y}\right\rangle \\
& =\frac{N k_{B} T}{\tau^{2}}\left\langle\left(\Delta \xi_{y}\right)^{2}\right\rangle,
\end{aligned}
$$

to the shear viscosity. There might be additional $1 / M$ corrections arising from the cell structure, but they must be proportional to some power of $a / \lambda$, since they must vanish in the continuum limit, $a / \lambda \rightarrow 0$. We will therefore neglect them for the moment. Using the large mean free path limit, Eq. (30), for $\left\langle\left(\Delta \xi_{y}\right)^{2}\right\rangle$ in Eq. (50), it follows that there is an additional contribution to expression (11) for the shear viscosity derived in the continuum limit in Sec. II A, so that

$$
\nu=\frac{k_{B} T \tau}{2}\left(\frac{1}{(1-1 / M) \sin ^{2}(\alpha)}-1\right)+\frac{a^{2}}{12 \tau} .
$$

For $\alpha=90^{\circ}$, this equation can be written as

$$
\nu=\frac{k_{B} T \tau}{2}\left\{\frac{1}{M-1}+\frac{1}{6}\left(\frac{a}{\lambda}\right)^{2}\right\},
$$

so that at finite $a / \lambda$, corrections are important for

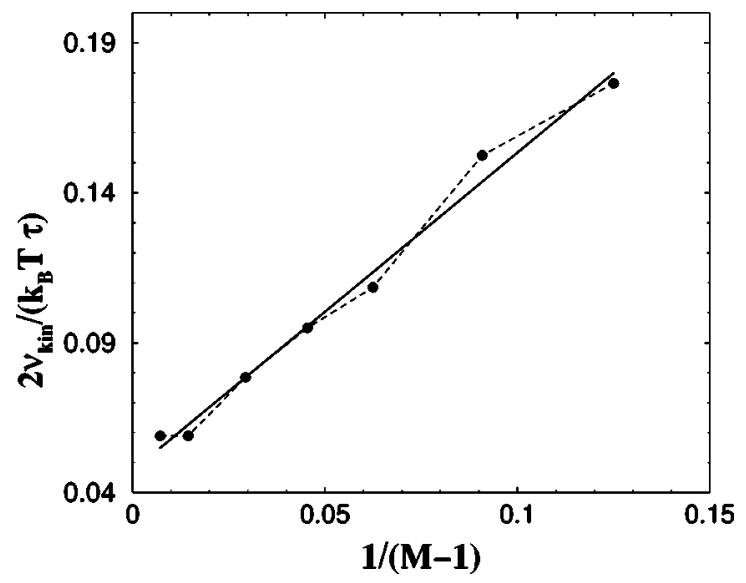

FIG. 4. The dimensionless kinetic contribution to the viscosity, $2 \nu_{k i n} /\left(k_{B} T \tau\right)$, as a function of $1 /(M-1)$ for $\alpha=90^{\circ}$. The symbols are simulation data, and the solid line is a fit with $1.06 /(M-1)$ +0.0473 . The agreement with the predicted behavior, Eq. (52), is excellent. Parameters: $\lambda / a=2, L=64, k_{B} T=4$.

$$
M>1+6(\lambda / a)^{2} .
$$

In most applications in two dimensions, $\alpha=90^{\circ}, \lambda / a \gtrsim 1$, and $5 \leqq M \leqq 50$, so that this correction is practically never negligible. Note, however, that this is true only in two dimensions and for $\alpha \approx 90^{\circ}$, where kinetic stress correlations oscillate and decay extremely slowly. It was previously believed that high Reynolds numbers could be achieved in this limit by choosing a large value of $\mathrm{M}$ in order to have a small viscosity. It is now clear, however, that this is problematic for two reasons. First, the minimum value for the viscosity is $a^{2} /(12 \tau)$, and not zero, for $M \rightarrow \infty$, and second, this is a pathological limit since the kinetic stress correlations oscillate in sign and do not decay.

In order to verify the dependence on $1 /(M-1)$ in Eq. (52), we performed a series of simulations at fixed large mean free path, $\lambda / a=2$, and plotted the kinetic part of the viscosity as a function of $1 /(M-1)$. The result is indeed a linear curve, Fig. 4 , which can be fitted by $2 \nu_{k i n} /\left(k_{B} T \tau\right)$ $=1.06 /(M-1)+0.0473$. Equation (52) predicts $1 /(M-1)$ +0.0417 , which is very close. Consider now the effect of a finite mean free path at fixed $M$. In Fig. 5, $\nu_{k i n}$ is plotted as a function of $\lambda / a$. In particular, we want to investigate whether the second term in Eq. (52) is indeed proportional to $(\lambda / a)^{2}$. The fit in Fig. 5 gives $(1 / 5.141)(\lambda / a)^{1.9988}$ for this term, which is again in good agreement.

Figure 6 shows measurements of the normalized viscosity at large and small mean free paths as a function of the rotation angle. It can be seen that Eq. (51) is in excellent agreement with the data around $90^{\circ}$. In contrast, without the correction term, the agreement is much worse; see Ref. [3]. Figure 7 shows the total viscosity as a function of mean free path.

\section{Calculation of $\left\langle\Delta \xi_{i y}^{(0)} v_{i x}^{(0)} \Delta \xi_{i y}^{(1)} v_{i x}^{(1)}\right\rangle$ at arbitrary angle, $\lambda / a \ll 1$}

Consider again the limit $M \rightarrow \infty$, but now for arbitrary scattering angle $\alpha$, so that collisions are described by Eqs. 


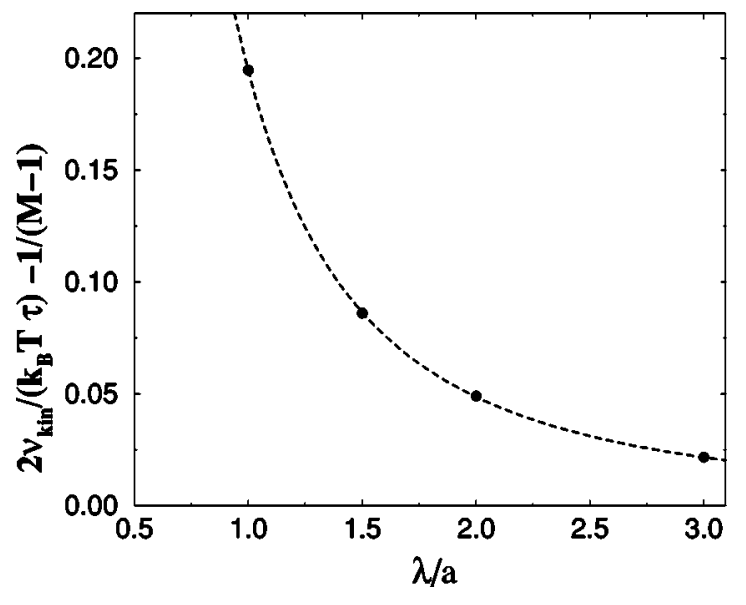

FIG. 5. $2 \nu_{k i n} /\left(k_{B} T \tau\right)-1 /(M-1)$ as a function of $\lambda / a$ for $\alpha$ $=90^{\circ}$. Symbols are simulation data, and the dashed line is the fit $0.1945(\lambda / a)^{\phi}$, with $\phi=-1.9988$. There is good agreement with the predicted $(\lambda / a)^{2} / 6$ behavior. Parameters: $M=35, L=128$.

(37) and (38). Only the limit $\lambda / a \ll 1$ will be considered in this section, so that $\Delta \xi_{y}$ can only take the values $-a, 0$, and $a$. The case of arbitrary mean free path will be considered elsewhere [5].

For $\lambda / a \ll 1$, there are only two contributions to $C_{1}$ $=\left\langle\Delta \xi_{i y}^{(0)} v_{i x}^{(0)} \Delta \xi_{i y}^{(1)} v_{i x}^{(1)}\right\rangle$. In the first, which we denote by $A_{1,-1}$ one has $\Delta \xi_{i x}^{(0)}=a$ and $\Delta \xi_{i x}^{(1)}=-a$; in the second, $A_{-1,1}, \Delta \xi_{i x}^{(0)}=-a$ and $\Delta \xi_{i x}^{(1)}=a$. Other possibilities are either identically zero-if one of the $\Delta \xi$ is zero-or occur with an exponentially small probability $\left[\sim \exp \left(-a^{2} / \lambda^{2}\right)\right]$, since they involve particles traveling distances of the order of $a$.

Consider first $A_{1,-1}$. In order for $\Delta \xi_{y}^{(0)}$ to be equal to $a$, $v_{y}^{(0)}$ must be positive; because $\lambda / a \ll 1$, it is also less than $a / \tau$. Similarly, in order that $\Delta \xi_{y}^{(1)}=-a$, we need $-a / \tau$ $<v_{y}^{(1)}<0$. A particle with $\left|v_{y}^{(0)}\right| \geqslant\left|v_{y}^{(1)}\right|$ will contribute to $A_{1,-1}$ if it is located in the interval $\left(y_{L} \equiv a-v_{y}^{(0)} \tau, y_{U} \equiv y_{L}\right.$

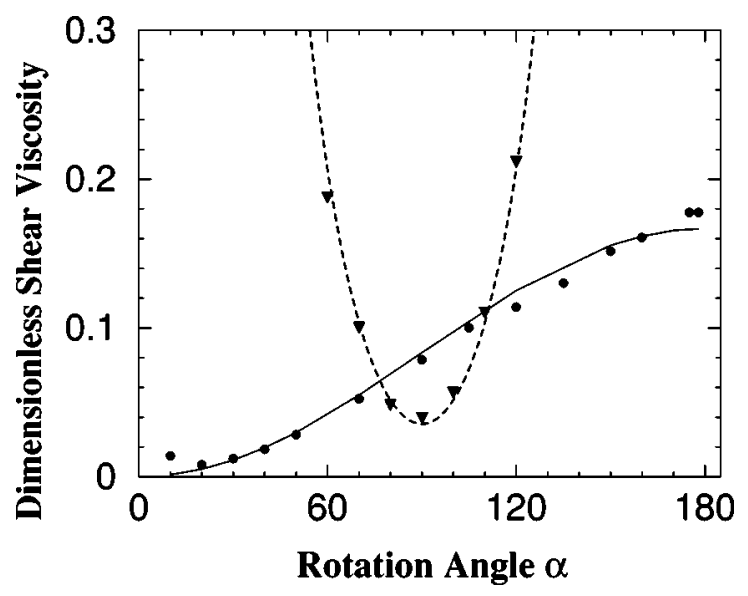

FIG. 6. Dimensionless shear viscosity measured at short times as a function of the rotation angle $\alpha$. The bullets are results for $\nu \tau / a^{2}$ at the small mean free path, $\lambda / a=0.028$. The solid line is a plot of Eq. (71). The inverted triangles are data for $\nu /\left(k_{B} T \tau\right)$ at large mean free path, $\lambda / a=2$. The dashed line is a plot of Eq. (51). The system size is $64 \times 64$ and $M=35$.

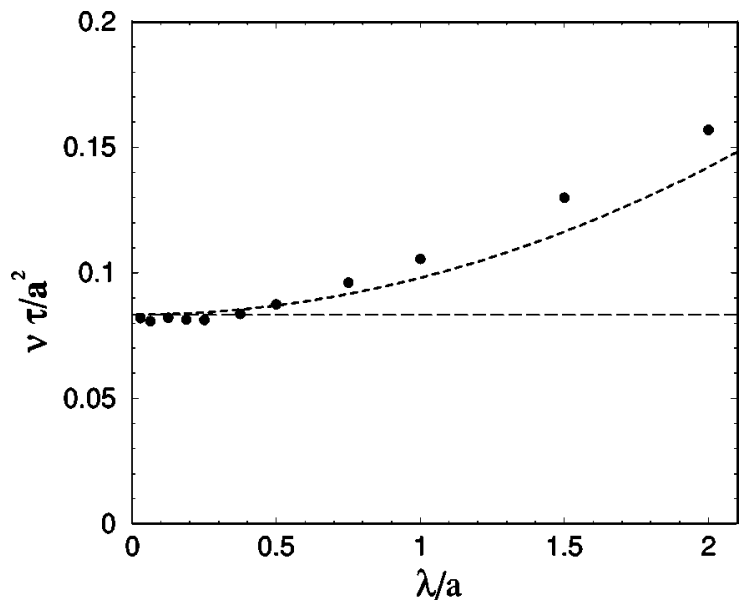

FIG. 7. Dimensionless shear viscosity measured at short times as a function of the mean free path. The bullets are simulation results for $\nu \tau / a^{2}$. The dashed line is the theoretical prediction for the kinetic part of the shear viscosity, given by Eq. (52). Parameters: $M=35, \alpha=90^{\circ}, L=64$.

$\left.-v_{y}^{(1)} \tau\right)$. Similarly, if $\left|v_{y}^{(0)}\right| \leqslant\left|v_{y}^{(1)}\right|$, it contributes only if it is located in the interval $\left(y_{L}=a-v_{y}^{(0)} \tau, y_{U}=a\right)$. The probability that a particle in a given cell contributes to $A_{1,-1}$ is therefore

$$
p_{1,-1}=\frac{\tau}{a} \min \left(v_{y}^{(0)},-v_{y}^{(1)}\right) .
$$

Using Eqs. (37) and (38), and the restrictions $0<v_{y}^{(0)}<a / \tau$ and $-a / \tau<v_{y}^{(1)}<0$, we have

$$
\begin{aligned}
A_{1,-1}= & -a^{2} \int_{0}^{a / \tau} d v_{y} \int_{d_{0}}^{d_{1}} p_{1,-1} d v_{x} v_{x}\left[c v_{x}\right. \\
& \left.+s v_{y}\right] w\left(v_{x}\right) w\left(v_{y}\right)
\end{aligned}
$$

where superscripts (0) have been dropped. Equation (55) is written for $z=1$; the final result derived below is the same for $z=-1$. The limits on the integral are

$$
d_{0}=c v_{y} / s
$$

and

$$
d_{1}=\left(a / \tau+c v_{y}\right) / s,
$$

where, as before, $c$ and $s$ are the cosine and the sine of the rotation angle $\alpha$, respectively. Finally, if Eq. (54) is used, we find

$$
\begin{aligned}
A_{1,-1}= & -a \tau \int_{0}^{a / \tau} w\left(v_{y}\right) d v_{y}\left\{\int _ { d _ { 0 } } ^ { d _ { 2 } } w ( v _ { x } ) v _ { x } \left[v_{x} v_{y}\left(s^{2}-c^{2}\right)\right.\right. \\
& \left.+c s\left(v_{x}^{2}-v_{y}^{2}\right)\right] d v_{x}+\int_{d_{2}}^{d_{1}} w\left(v_{x}\right) v_{x} v_{y} \\
& \left.\times\left[c v_{x}+s v_{y}\right] d v_{x}\right\}
\end{aligned}
$$


with $d_{2}=(1+c) v_{y} / s$. For symmetry reasons, the other contribution to $C_{1}$, namely, $A_{-1,1}$ is equal to $A_{1,-1}$. Evaluating the integrals, we have

$$
C_{1}=-\frac{k_{B} T a \lambda}{\sqrt{\pi}}\left[c \sqrt{2}+\frac{\sqrt{1+c}}{2}(1-3 c)\right],
$$

so that

$$
\begin{gathered}
C_{1}=0 \quad \text { for } \alpha=0^{\circ}, \\
C_{1}=-\frac{k_{B} T a \lambda}{\sqrt{\pi}}\left[\frac{1}{2}+c\left(\sqrt{2}-\frac{5}{4}\right)\right] \text { for } \alpha \approx 90^{\circ}, \\
C_{1}=k_{B} T a \lambda \sqrt{2 / \pi} \text { for } \alpha=180^{\circ} .
\end{gathered}
$$

Equation (61) agrees with the small $\lambda$ limit of Eq. (47). Simple arguments can be used to show that the limits $\alpha$ $=0^{\circ}$ and $180^{\circ}$ are also correct.

Figure 3(b) shows the angular dependence of the ratio $g$ $=C_{1} /\left\langle\left(\Delta \xi_{y} v_{x}\right)^{2}\right\rangle$, which describes the initial decay of the stress correlations. The behavior at small mean free path is clearly qualitatively different than that for large $\lambda$. In particular, the symmetry around $\alpha=90^{\circ}$ is lost at small $\lambda$.

\section{ANALYSIS OF THE ROTATIONAL AND MIXED CONTRIBUTIONS TO THE SHEAR VISCOSITY}

\section{A. Simple approximation for the rotational contribution}

Since the particle collisions occur in a shifted cell coordinate system, they result in a transfer of momentum between neighboring cells in the original unshifted reference frame. For $\lambda / a \ll 1$, this rotational contribution is much larger than the kinetic contribution and determines the value of shear viscosity. Simple kinetic arguments can be used to obtain a surprisingly accurate expression for this rotational contribution to the shear viscosity. Consider a collision cell of size $a \times a$ oriented parallel to the $x$ and $y$ axes, and divide the cell by the line $y=h$. The line $y=h$ represents the boundary between cells in the original unshifted reference frame. Since we consider only momentum transfer in the $y$ direction, this one-dimensional shift operation is sufficient. Assuming a homogeneous particle distribution, the upper part of the cell contains $M_{1}=M(a-h) / a$ particles on an average, while the lower part has $M_{2}=M h / a$ particles, with $M_{1}+M_{2}=M$. The mean velocities $\mathbf{u}_{1}$ and $\mathbf{u}_{2}$ in the upper and lower partitions are

$$
\mathbf{u}_{1}=\frac{1}{M_{1}} \sum_{i=1}^{M_{1}} \mathbf{v}_{i}
$$

and

$$
\mathbf{u}_{2}=\frac{1}{M_{2}} \sum_{i=M_{1}+1}^{M} \mathbf{v}_{i}
$$

respectively. Since the mean free path is assumed to be very small, the definition of mean velocities on scales smaller than a cell size is justified. The stochastic rotation of velocities in the collision step transfers momentum between the two parts of the cell. The $x$ component of this momentum transfer is

$$
\Delta p_{x}(h) \equiv \sum_{i=1}^{M_{1}}\left[v_{i, x}(t+\tau)-v_{i, x}(t)\right]
$$

Averaging now over the sign of the stochastic rotation angle, we have

$$
\Delta p_{x}(h)=(1-c) M_{1}\left(u_{x}-u_{1, x}\right)
$$

where we used the evolution equation (2), with $c=\cos (\alpha)$. The $y$ component of the velocity does not appear in this expression because the average of the sine of the rotation angle is zero. Using $M \mathbf{u}=M_{1} \mathbf{u}_{1}+M_{2} \mathbf{u}_{2}$, the difference $u_{x}$ $-u_{1, x}$ can be rewritten as $M_{2}\left(u_{2, x}-u_{1, x}\right) / M$, so that

$$
\Delta p_{x}(h)=(1-c) M\left(u_{2, x}-u_{1, x}\right) \frac{h}{a}\left(1-\frac{h}{a}\right) .
$$

Averaging over the position $h$ of the dividing line corresponds to averaging over the random shift of the grid. Doing this, we have

$$
\left\langle\Delta p_{x}\right\rangle=\frac{1}{a} \int_{0}^{a} \Delta p_{x}(h) d h=\frac{1}{6}(1-c) M\left(u_{2, x}-u_{1, x}\right) .
$$

Finally, if we approximate $\partial u_{x} / \partial y$ by

$$
\frac{\partial u_{x}}{\partial y}=\left(u_{1, x}-u_{2, x}\right) /(a / 2),
$$

the shear viscosity $\eta$, which is defined as the ratio of the tangential stress $P_{y x}$ to $\partial u_{x} / \partial y$, can be expressed as

$$
\eta=\frac{P_{y x}}{\partial u_{x} / \partial y}=\frac{\left\langle\Delta p_{x}\right\rangle /(a \tau)}{\partial u_{x} / \partial y}
$$

since the tangential stress $P_{y x}$ is the mean increase, per unit time and unit length, of the $x$ component of the momentum of the gas across the line $y=h$. Using Eqs. (68) and (69) in Eq. (70), we obtain the result

$$
\nu=\frac{a^{2}}{12 \tau}[1-\cos (\alpha)]
$$

for the kinematic viscosity $\nu$ in the limit of small mean free path. We checked the dependence of the viscosity on the rotation angle, Fig. 6, time step, temperature, and cell size, Fig. 7, and found very good agreement between the simulations and Eq. (71) in this limit. Although this derivation is somewhat heuristic, it gives the prefactor with an unexpected accuracy, as can be seen in Fig. 6. 

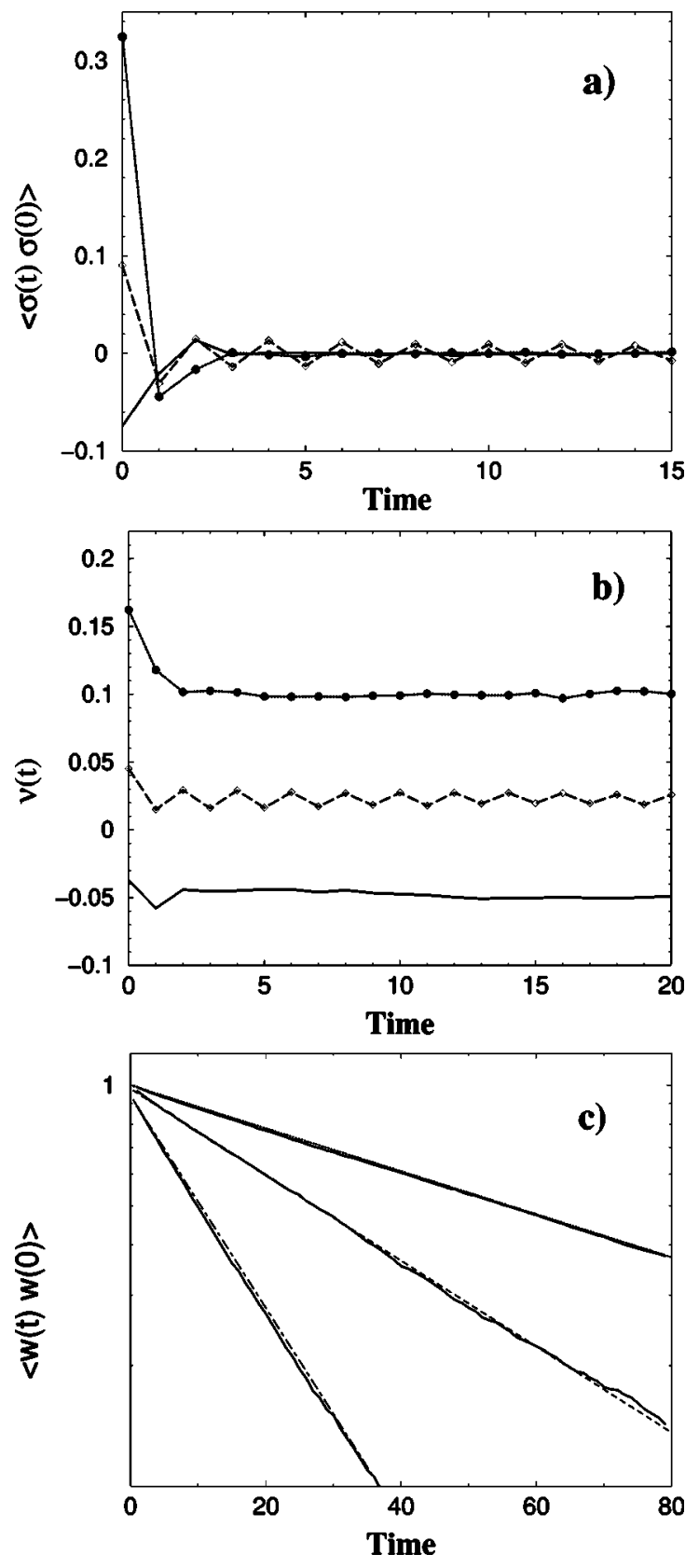

FIG. 8. Simulation results for small mean free path, $\lambda / a$ $=0.113$, and $\alpha=90^{\circ}$. (a) Transverse stress correlations as a function of time. Dotted line $(\bullet)$, rotational part $\left\langle\sigma_{r o t}(t) \sigma_{r o t}(0)\right\rangle$; dashed line $(\diamond)$, kinetic part $\left\langle\sigma_{k i n}(t) \sigma_{k i n}(0)\right\rangle$; solid line, mixed part $\left\langle\sigma_{k i n}(t) \sigma_{\text {rot }}(0)\right\rangle+\left\langle\sigma_{\text {rot }}(t) \sigma_{\text {kin }}(0)\right\rangle$. (b) Various contributions to the shear viscosity obtained by summing the stress correlation functions from (a). Dotted line (@), rotational viscosity $\nu_{\text {rot }}$; dashed line $(\diamond)$, kinetic viscosity $\nu_{k i n}$; solid line, contribution of the mixed term to the viscosity, $\nu_{m i x}$. (c) Vorticity correlations $\left\langle w_{k}(t) w_{-k}(0)\right\rangle$ as a function of time for three different wave numbers $k$ (thick solid curves). The thin dashed-dotted lines are fits with the function $\exp \left(-\nu_{F i t} k^{2} t\right)$. The fitted $k$-dependent viscosities $\nu_{F i t}$ are upper line, $k=0.3927, \nu_{\text {Fit }}=0.08$; middle line, $k=0.55536$, $\nu_{F i t}=0.08$; lowest line, $k=0.8781, \nu_{F i t}=0.079$. Parameters: $M$ $=35, L=16, \tau=1$; time average over 30000 iteration steps and two different initial conditions.

\section{B. Calculation of rotational and mixed stress correlations at equal time}

We learned from simulations that the temporal correlations of the rotational and the mixed part of the stress tensor usually decay very rapidly. Hence, the significant contributions to the rotational and the mixed viscosity come from the first two to four terms in the sum in Eq. (1). We will concentrate only on the first term, the correlations at equal time, and compare them to the numerical values.

\section{Rotational term}

The $t=0$ contribution to the rotational part of the shear viscosity is

$$
\nu_{r o t}(0)=\left\langle\sigma_{r o t}^{2}(0)\right\rangle /\left(2 N k_{B} T \tau\right),
$$

with

$$
\left\langle\sigma_{r o t}^{2}(0)\right\rangle=\sum_{i, j}^{N}\left\langle\Delta v_{i y} \Delta \xi_{i x}^{s} \Delta v_{j y} \Delta \xi_{j x}^{s}\right\rangle .
$$

In contrast to the calculation of the kinetic stress correlations, off-diagonal contributions to Eq. (73) are not negligible, even for large $M$, and we have to adopt a different approach.

Although the following arguments are easily generalized, consider a specific random shift vector $\mathbf{b}=(b, 0)$ with $b$ $\in[0, a / 2)$. In this case $\Delta \xi_{i x}^{s}=-a$ for all particles located in a strip of width $b$ located on the right edge of a cell. The average number of particles in this strip is $P=b M / a$. For all other particles in the cell, $\Delta \xi_{i x}^{s}=0$. This means that in every cell, only $P$ particles contribute to $\left\langle\sigma_{\text {rot }}^{2}\right\rangle$, and we can simplify expression (73). Averaging over all possible shifts leads to

$$
\left\langle\sigma_{r o t}^{2}(0)\right\rangle=2 a L^{2} \int_{0}^{a / 2} d b\left\langle\left(\sum_{i}^{P} \Delta v_{i y}\right)^{2}\right\rangle
$$

where the sum runs over all particles located in the strip at the right edge of the cell. $L^{2}$ is the total number of cells. Using Eq. (3) for the stochastic rotation of the $y$ component of the velocity, one finds

$$
\begin{aligned}
\sum_{i}^{P} \Delta v_{i y}= & (1-c) \frac{P}{M} \sum_{i}^{M} v_{i y}+(c-1) \sum_{i}^{P} v_{i y}-s \sum_{i}^{P} v_{i x} \\
& +s \frac{P}{M} \sum_{i}^{M} v_{i x},
\end{aligned}
$$

where $\sum_{i}^{M}$ is the sum over all particles in the cell (in the shifted system), and $\Sigma_{i}^{P}$ is the sum over the particles in the strip. $c$ and $s$ are the cosine and sine of the rotation angle, respectively. Assuming ideal gas correlations $\left\langle v_{i \alpha} v_{j \beta}\right\rangle$ $=\delta_{i j} \delta_{\alpha \beta} k_{B} T$, one obtains

$$
\left\langle\left(\sum_{i}^{P} \Delta v_{i y}\right)^{2}\right\rangle=2(1-c) P\left(1-\frac{P}{M}\right) k_{B} T .
$$



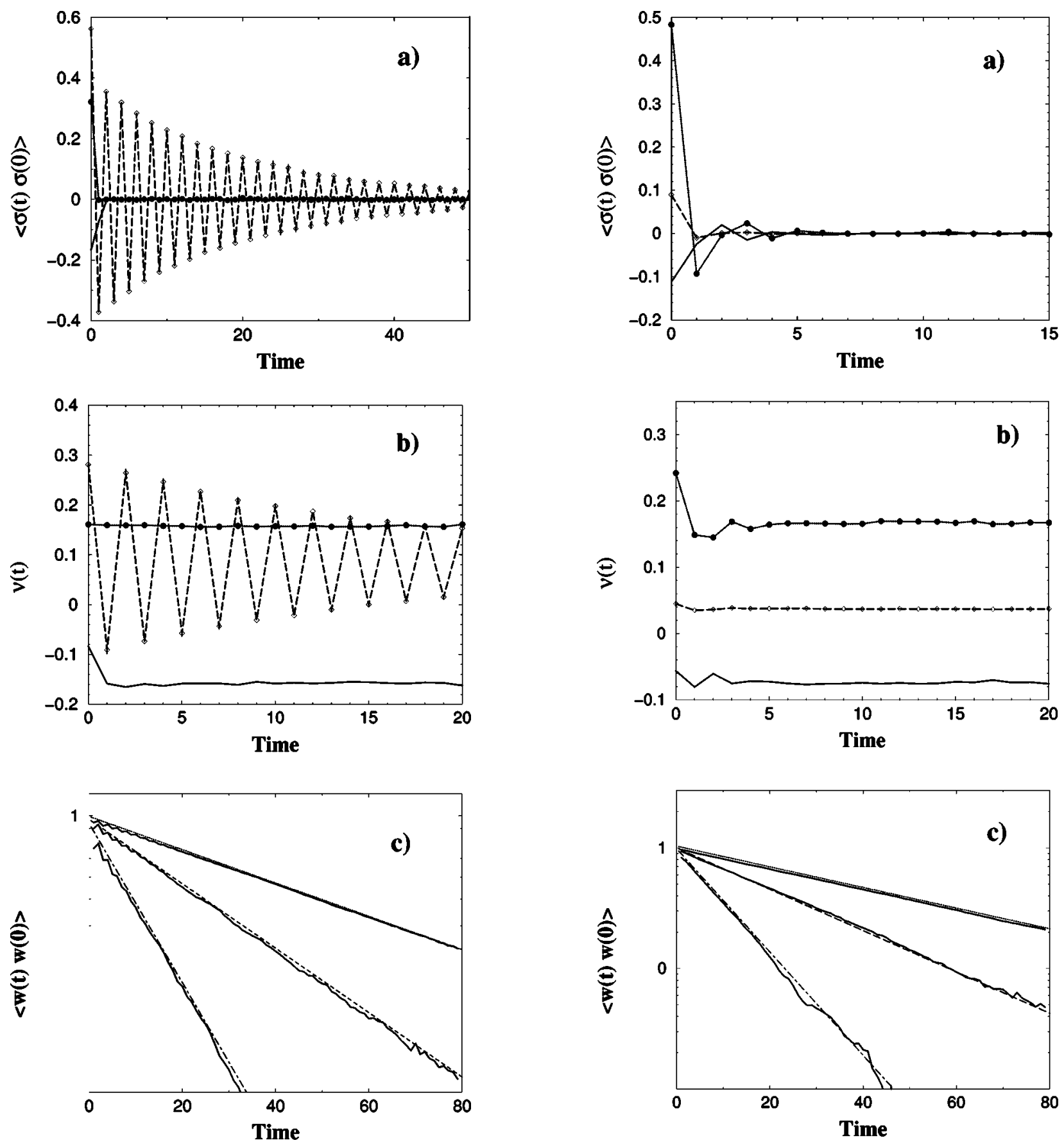

FIG. 9. Simulations results for intermediate mean free path, $\lambda / a=0.632$, and $\alpha=90^{\circ}$. (a) Transverse stress correlations as a function of time. Dotted line $(\mathbf{O})$, rotational part $\left\langle\sigma_{r o t}(t) \sigma_{r o t}(0)\right\rangle$; dashed line $(\diamond)$, kinetic part $\left\langle\sigma_{\text {kin }}(t) \sigma_{\text {kin }}(0)\right\rangle$; solid line, mixed part $\left\langle\sigma_{\text {kin }}(t) \sigma_{\text {rot }}(0)\right\rangle+\left\langle\sigma_{\text {rot }}(t) \sigma_{\text {kin }}(0)\right\rangle$. (b) Various contributions to the shear viscosity obtained by summing the stress correlation functions from (a). Dotted line $(\mathbf{O})$, rotational viscosity $\nu_{\text {rot }}$; dashed line $(\diamond)$, kinetic viscosity $\nu_{k i n}$; solid line, contribution of the mixed term to the viscosity $\nu_{m i x}$. (c) Vorticity correlations $\left\langle w_{k}(t) w_{-k}(0)\right\rangle$ as a function of time for three different wave numbers $k$ (thick solid curves). The thin dashed-dotted lines are fits with the function $\exp \left(-\nu_{F i t} k^{2} t\right)$. The fitted $k$-dependent viscosities $\nu_{F i t}$ are upper line, $k=0.3927, \nu_{F i t}=0.087$; middle line, $k=0.55536$, $\nu_{F i t}=0.087$; lowest line, $k=0.8781, \nu_{F i t}=0.086$. Parameters: $M$ $=35, L=16, \tau=1$.

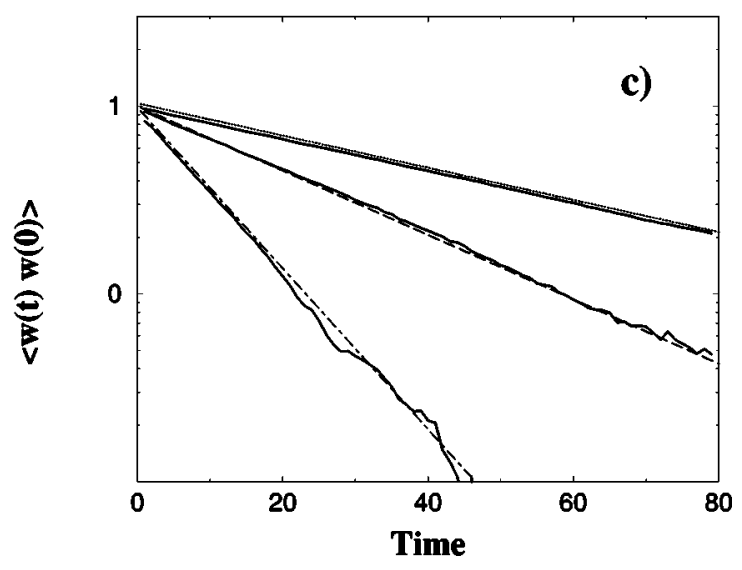

FIG. 10. Simulation results for small mean free path, $\lambda / a$ $=0.113$, and $\alpha=120^{\circ}$. (a) Transverse stress correlations as a function of time. Dotted line $(\circlearrowleft)$, rotational part $\left\langle\sigma_{r o t}(t) \sigma_{r o t}(0)\right\rangle$; dashed line $(\diamond)$, kinetic part $\left\langle\sigma_{k i n}(t) \sigma_{k i n}(0)\right\rangle$; solid line, mixed part $\left\langle\sigma_{k i n}(t) \sigma_{\text {rot }}(0)\right\rangle+\left\langle\sigma_{\text {rot }}(t) \sigma_{k i n}(0)\right\rangle$. (b) Various contributions to the shear viscosity obtained by summing the stress correlation functions from (a). Dotted line $(\mathbf{O})$, rotational viscosity $\nu_{r o t}$; dashed line $(\diamond)$, kinetic viscosity $\nu_{k i n}$; solid line, contribution of the mixed term to the viscosity $\nu_{m i x}$. (c) Vorticity correlations $\left\langle w_{k}(t) w_{-k}(0)\right\rangle$ as a function of time for three different wave numbers $k$ (thick solid curves). The thin dashed-dotted lines are fits with the function $\exp \left(-\nu_{F i t} k^{2} t\right)$. The fitted $k$-dependent viscosities $\nu_{F i t}$ are upper line, $k=0.3927, \nu_{F i t}=0.128$; middle line, $k=0.55536$, $\nu_{F i t}=0.128$; lowest line, $k=0.8781, \nu_{F i t}=0.128$. Parameters: $M$ $=35, L=16, \tau=1$. 
Inserting $P=b M / a$ and integrating over $b$ yields

$$
\left\langle\sigma_{r o t}^{2}\right\rangle=\frac{a^{2}}{3}[1-\cos (\alpha)] N k_{B} T,
$$

so that we have, finally,

$$
\nu_{r o t}(0)=\left\langle\sigma_{r o t}^{2}(0)\right\rangle /\left(2 N k_{B} T \tau\right)=\frac{a^{2}}{6 \tau}[1-\cos (\alpha)] .
$$

\section{Mixed term}

The equal time contribution of the mixed term to the total viscosity is

$$
C_{m i x}=2\left\langle\sigma_{r o t}(0) \sigma_{k i n}(0)\right\rangle=2 \sum_{i, j}^{N}\left\langle\Delta \xi_{i x} v_{i y} \Delta \xi_{j x}^{s} \Delta v_{j y}\right\rangle .
$$

Here we will make the assumption that only the diagonal terms contribute, i.e.,

$$
C_{m i x} \approx 2 N\left\langle v_{i y}^{(0)}\left(v_{i y}^{(1)}-v_{i y}^{(0)}\right)\right\rangle\left\langle\Delta \xi_{i x} \Delta \xi_{i x}^{s}\right\rangle .
$$

Using expression (36), and the evolution equation for the velocities, Eqs. (2) and (3), we find

$$
\begin{aligned}
C_{\text {mix }} \approx & -a^{2}[1-\cos (\alpha)] N k_{B} T\left[\frac{1}{6}-\frac{1}{\pi^{2}} \sum_{n=1}^{\infty} \frac{1}{n^{2}}\right. \\
& \left.\times \exp \left\{-2 \pi^{2} n^{2}\left(\frac{\lambda}{a}\right)^{2}\right\}\right] .
\end{aligned}
$$

The zeroth-order contribution to the viscosity is therefore

$$
\begin{aligned}
\nu_{\text {mix }}(0) \approx & \frac{C_{\text {mix }}}{2 N k_{B} T \tau}=\frac{a^{2}}{2 \Delta t}[1-\cos (\alpha)]\left[\frac{1}{6}-\frac{1}{\pi^{2}}\right. \\
& \left.\times \sum_{n=1}^{\infty} \frac{1}{n^{2}} \exp \left\{-2 \pi^{2} n^{2}\left(\frac{\lambda}{a}\right)^{2}\right\}\right]
\end{aligned}
$$

In the limit $\lambda \ll a$ this gives

$$
\nu_{\text {mix }}(0) \approx \frac{a^{2}}{\sqrt{2 \pi} \tau}[1-\cos (\alpha)] \frac{\lambda}{a}
$$

\section{Comparison to simulations}

A set of simulations was performed to evaluate the various contributions to the stress correlations and to measure the transport coefficients. The results are presented in Figs. 8-11. After a short equilibration time, averages were typically performed over 30000-60000 time steps. For simulations with $N=20000-150000$ particles and system size $L$ $=64$ or 128 , the CPU time per time step and per particle was between 1.8 and $2.3 \mu$ s on an IBM SP (serial code, $375 \mathrm{MHz}$ Power3 processor), depending on whether the cell shift was implemented or not, or whether the rotation angle was $90^{\circ}$ (the computationally most efficient value). This CPU time estimate includes the overhead caused by the evaluation of the transport coefficients, and would therefore be smaller for real production runs. For systems with a larger number of particles, $N=3 \times 10^{5}-6 \times 10^{5}$, the average CPU time per iteration and per particle increased to about $3.3 \mu \mathrm{s}$.

Note that $\nu_{r o t}(0)$, Eq. (78), is up to a factor of 2 larger than the viscosity measured at small mean free path, Eq. (71). This means that the contribution from the next term, $\left\langle\sigma_{\text {rot }}(\tau) \sigma_{\text {rot }}(0)\right\rangle$, is not negligible (for $\lambda \ll a$ ) and must be negative. This is clearly visible in our simulations, see Fig. 8.

For large mean free path, see Fig. 9, $\left\langle\sigma_{\text {rot }}(\tau) \sigma_{\text {rot }}(0)\right\rangle$ was found to be approximately zero, i.e., $\nu(0)$ is approximately equal to $\nu_{\text {rot }}$. In Fig. 9(a), it can be seen that the decay rate of the kinetic stress correlations is larger from $t$ $=0$ to $t=\tau$ than for larger values of $t$. This is consistent with Eqs. (48) and (49). Plotting the data in Fig. 9(a) on a logarithmic scale (not shown) confirms the hypothesis made following Eq. (48) that the ratio of higher-order terms such as $C_{2} / C_{1}, C_{3} / C_{2}$, etc., is constant and given by the continuum approximation. $g=C_{1} / C_{0}$, on the other hand, contains finite $a$ correction terms.

Figure 10 shows the temporal behavior for the kinetic, rotational, and mixed stress correlations for an equilibrium system with $\alpha=120^{\circ}, M=35$, and $\lambda / a=0.1129$. We measured the values $h\left\langle\sigma_{\text {rot }}^{2}(0)\right\rangle=0.481, h\left\langle\sigma_{\text {rot }}(0) \sigma_{\text {kin }}(0)\right\rangle=$ -0.056 , and $h\left\langle\sigma_{\text {kin }}^{2}(0)\right\rangle=0.09$, where $h=\tau /\left(a^{2} N k_{B} T\right)$ is a normalization factor. These values are generally in good agreement with the theoretical results obtained from Eqs. (77), (81), (28), and (50), namely, $h\left\langle\sigma_{\text {rot }}^{2}(0)\right\rangle_{\text {theo }}=0.5$, $h\left\langle\sigma_{\text {rot }}(0) \sigma_{\text {kin }}(0)\right\rangle_{\text {theo }}=-0.0675, \quad$ and $h\left\langle\sigma_{\text {kin }}^{2}(0)\right\rangle_{\text {theo }}$ $=0.09$. The largest discrepancy is for the mixed contribution, which is overestimated by about $20 \%$. This is not surprising, however, since we neglected the off-diagonal contribution in the derivation of Eq. (81).

For larger mean free path, $\lambda / a=0.632$ and $\alpha=90^{\circ}$, see Fig. 9, the agreement is very good for all three quantities. We found $h\left\langle\sigma_{\text {rot }}^{2}(0)\right\rangle=0.32$ (theory, 0.33), $h\left\langle\sigma_{\text {rot }}(0) \sigma_{\text {kin }}(0)\right\rangle$ $=-0.084$ (theory, 0.0833), and $h\left\langle\sigma_{\text {kin }}^{2}(0)\right\rangle=0.565$ (theory, $0.564)$.

\section{Cancellation of errors}

Figure 11(b) shows the various contributions to the time dependent viscosity (i.e., the integrated stress correlations up to time $t$ ) for $\alpha=60^{\circ}, M=35$, and $\lambda / a=0.1129$. The heuristic derivation of the rotational contribution to the viscosity given in Sec. III A, Eq. (71), predicts a value 0.0416 for the viscosity which is in excellent agreement with the measured total viscosity 0.042 . The value we get for the rotational contribution from the Green-Kubo formula, see Fig. 11(b), is 0.062 , i.e., is about $50 \%$ larger. This means that the contributions from the kinetic and the mixed part, which were not included in the derivation of Eq. (71), are equal to -0.02 , and not negligible, even at this small mean free path. It appears that this cancellation occurs for a wide range of rotation angles, between $30^{\circ}$ and $160^{\circ}$, for reasons we do not yet understand. 

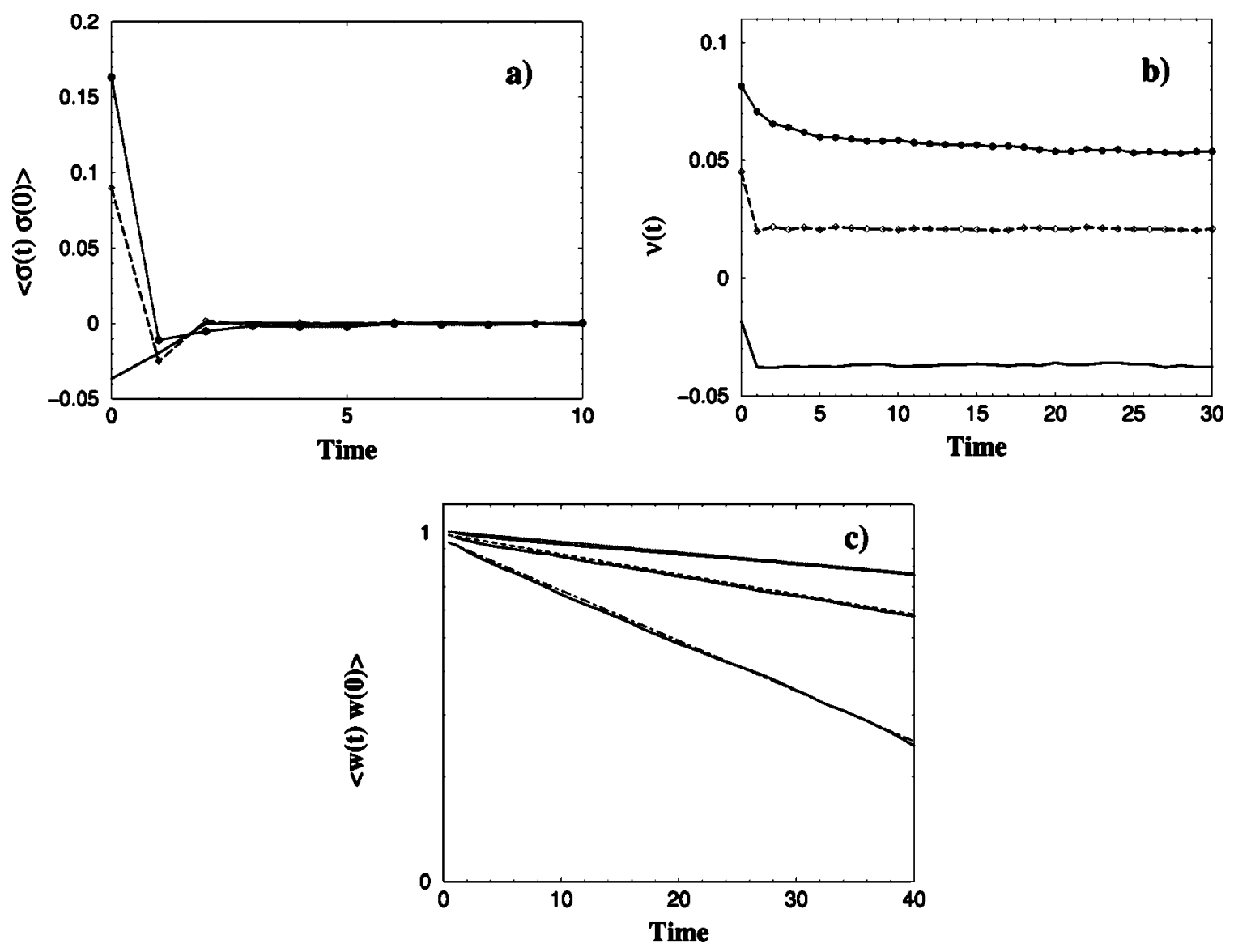

FIG. 11. Simulation results for small mean free path, $\lambda / a=0.113$, and $\alpha=60^{\circ}$. (a) Transverse stress correlations as a function of time. Dotted line $(\bigcirc)$, rotational part $\left\langle\sigma_{r o t}(t) \sigma_{r o t}(0)\right\rangle$; dashed line $(\diamond)$, kinetic part $\left\langle\sigma_{k i n}(t) \sigma_{k i n}(0)\right\rangle$; solid line, mixed part $\left\langle\sigma_{k i n}(t) \sigma_{r o t}(0)\right\rangle$ $+\left\langle\sigma_{\text {rot }}(t) \sigma_{\text {kin }}(0)\right\rangle$. (b) Various contributions to the shear viscosity obtained by summing the stress correlation functions from (a). Dotted line $(\bullet)$, rotational viscosity $\nu_{\text {rot }}$; dashed line $(\diamond)$, kinetic viscosity $\nu_{k i n}$; solid line, contribution of the mixed term to the viscosity $\nu_{m i x}$. (c) Vorticity correlations $\left\langle w_{k}(t) w_{-k}(0)\right\rangle$ as a function of time for three different wave numbers $k$ (thick solid curves). The thin dasheddotted lines are fits with the function $\exp \left(-\nu_{F i t} k^{2} t\right)$. The fitted $k$-dependent viscosities $\nu_{F i t}$ are upper line, $k=0.3927, \nu_{F i t}=0.045$; middle line, $k=0.55536, \nu_{F i t}=0.043$; lowest line, $k=0.8781, \nu_{F i t}=0.043$. Parameters: $M=35, L=16, \tau=1$. Here and in the following all times, lengths, and masses are given in internal units of the simulation. The internal units for viscosities and other quantities follow from those definitions. In those units the time step is equal to $\tau$, the mass is equal to 1 , and the lattice constant of the grid is equal to $a$.

\section{ANALYSIS OF THE BULK VISCOSITY}

\section{Continuum approximation, $\lambda / a \rightarrow \infty$}

The kinematic bulk viscosity $\gamma$ can be determined in a similar fashion. In particular, taking now $\hat{\mathbf{k}}$ in the $x$ direction and $\alpha=\beta=1$ in Eq. (54) of Part 1, we have

$$
\nu+\gamma=\frac{\tau}{N k_{B} T} \sum_{t=0}^{\infty}\left\langle I_{2}(\hat{x}, 0) \mid I_{2}(\hat{x}, t)\right\rangle .
$$

Employing the same approximations as in Sec. II A, one finds

$$
\left\langle I_{2}(\hat{x}, 0) \mid I_{2}(\hat{x}, n \tau)\right\rangle=N\left(k_{B} T\right)^{2}[\zeta+\eta]^{n} .
$$

This is the same result as that obtained in Sec. II A [see Eq. (10)] for $\left\langle I_{2}(\hat{y}, 0) \mid I_{2}(\hat{y}, t)\right\rangle$, the corresponding term in the Green-Kubo relation for the shear viscosity. It follows that the bulk viscosity is zero for this model. This is the same result as for an ideal gas. Measurements of the bulk viscosity via the Green-Kubo formula, see Fig. 12, confirm that the bulk viscosity is essentially zero and has no long-time tail.

\section{ANALYSIS OF THE THERMAL DIFFUSIVITY}

\section{A. Continuum approximation, $\lambda / a \rightarrow \infty$}

The Green-Kubo relation for the thermal transport coefficient is given in Eq. (57) of Part 1, and the corresponding flux in Eq. (55) of Part 1. In the limit of large mean free path, we can make the same approximations as in Sec. II A. In two dimensions, taking $\hat{\mathbf{k}}$ in the $x$ direction, we then have

$$
I_{d+2}(\hat{\mathbf{k}}, t)=\sum_{j=1}^{N}\left[v_{j}^{2}(t) / 2-c_{p} T\right] v_{j x}(t) .
$$

Defining $E_{n} \equiv\left\langle I_{d+2}(\hat{x}, 0) \mid I_{d+2}(\hat{x}, n \tau)\right\rangle$, and using the same analysis as in Sec. II A, one has

$$
E_{1}=2 N\left(k_{B} T\right)^{3}\left[\zeta_{1}\left(\zeta_{1}^{2}+\zeta_{2}^{2}\right)+\eta_{1}\right]
$$




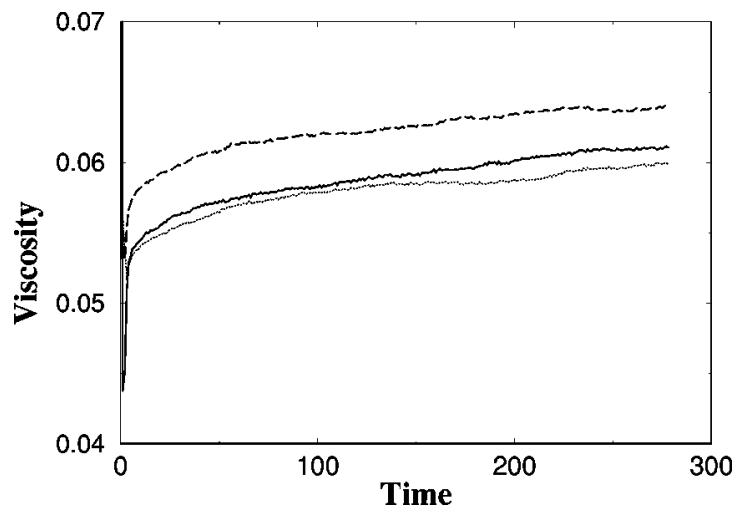

FIG. 12. Temporal behavior of bulk and shear viscosities determined from the Green-Kubo relations. The dashed line is the kinetic contribution to the sum of bulk and shear viscosities, $\nu_{k i n}+\gamma_{k i n}$, obtained from the longitudinal stress correlations. The solid line is the total measured bulk and shear viscosities, $\nu+\gamma$; the dotted line is the result for $\nu$ determined from the transverse stress correlations. The good agreement of these results indicates that the bulk viscosity $\gamma$ is negligibly small at all times. Parameters: $M=5, L=64, \alpha$ $=60^{\circ}, k_{B} T=0.0413, \lambda / a=0.203$; time average over 130000 time steps and over 40 different initial seeds of the random number generator.

where $\eta_{1}=2(M-1)(1-c)^{2} / M^{3}$ and $\zeta_{1}$ and $\zeta_{2}$ are defined in Sec. II A. The two terms in Eq. (87) are the diagonal and off-diagonal contributions, respectively. Similarly, one finds

$$
E_{n}=2 N\left(k_{B} T\right)^{3}\left[\zeta_{1}\left(\zeta_{1}^{2}+\zeta_{2}^{2}\right)+\eta_{1}\right]^{n}
$$

so that

$$
D_{T}=\frac{k_{B} T}{2} \tau\left[\frac{1+\zeta_{1}\left(\zeta_{1}^{2}+\zeta_{2}^{2}\right)+\eta_{1}}{1-\zeta_{1}\left(\zeta_{1}^{2}+\zeta_{2}^{2}\right)-\eta_{1}}\right]
$$

\section{B. Measurements}

Figure 13 shows the thermal diffusivity as a function of rotation angle. As can be seen, there is very good agreement with the theoretical prediction, Eq. (89). Figure 14 shows that the kinetic contribution to the heat diffusivity is much larger than the rotational contribution, even at small mean free path. Furthermore, it can be seen that $D_{T}$ diverges logarithmically with time, as predicted by mode-coupling theory $[6,7]$.

\section{Calculation of the rotational part of the thermal diffusivity}

As can be seen in Fig. 14, the rotational contribution to the thermal diffusivity, $D_{T, r o t}$, is small compared to the total diffusivity and can be neglected even at small mean free path $\lambda / a \sim 0.1$. Figure 14 also shows that that $D_{T, r o t}$ is essentially independent of time, which means that only the $t=0$ equal time correlations of the heat flux are nonzero. This contribution can be determined using the approach applied in Sec. III B 1 to calculate a similar contribution to the rotational part of the shear viscosity, Eq. (78).

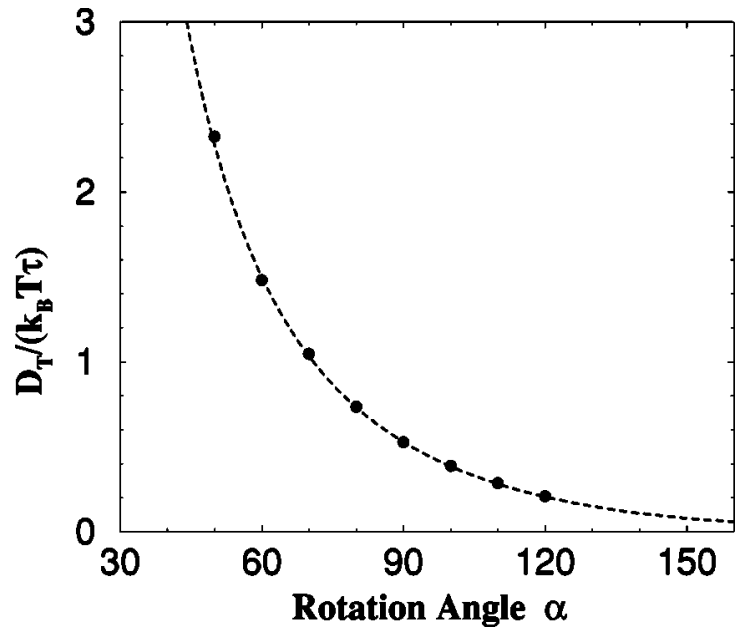

FIG. 13. Dimensionless thermal diffusivity $D_{T} /\left(k_{B} T \tau\right)$ at large mean free path, $\lambda / a=2$, as a function of the rotation angle $\alpha$. The dashed line is the theoretical prediction, Eq. (89). Parameters: $M$ $=35, k_{B} T=4, L=64$. Here and in the following all angles are measured in degrees.

The $t=0$ rotational contribution to the Green-Kubo relation for the thermal diffusivity [Eq. (51) of Part 1] in two dimensions is

$$
D_{T, r o t}(0)=\frac{1}{16 \tau N\left(k_{B} T\right)^{2}}\left\langle\sigma_{T, r o t}^{2}(0)\right\rangle,
$$

with

$$
\left\langle\sigma_{T, r o t}^{2}(0)\right\rangle=\sum_{i, j}^{N}\left\langle\Delta v_{i}^{2} \Delta \xi_{i x}^{s} \Delta v_{j}^{2} \Delta \xi_{j x}^{s}\right\rangle
$$

The approach of Sec. III B 1 can be used to show that

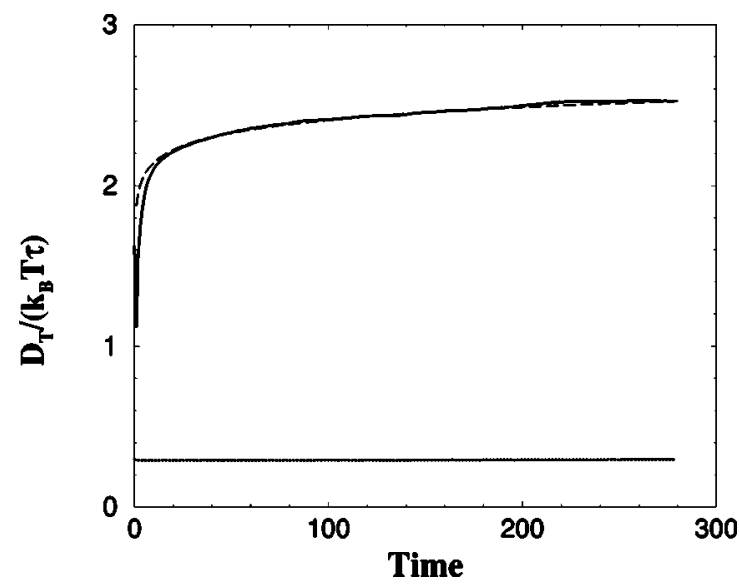

FIG. 14. Long-time tail of the dimensionless thermal diffusivity $D_{T} /\left(k_{B} T \tau\right)$ (solid curve) as a function of time for small $M=5$. The dashed line is the fit $1.876+0.115 \ln (t)$. The small deviation at $t$ $\approx 220$ agrees well with the recurrence time for sound waves, $t_{S}$ $=222.7$. The lower dotted line is the rotational part of the thermal diffusivity, $D_{T, r o t}$, which is predicted to be small; see Eq. (95). Parameters: $k_{B} T=0.0413, L=64, \lambda / a=0.203, \alpha=60^{\circ}$. 


$$
\left\langle\sigma_{T, r o t}^{2}(0)\right\rangle=2 a L^{2} \int_{0}^{a / 2} d b\left\langle\left(\sum_{i=1}^{P} \Delta v_{i}^{2}\right)^{2}\right\rangle,
$$

where $\Delta v_{i}^{2}=v_{i}^{2}(\tau)-v_{i}^{2}(0)$, and the sum goes over all $P$ $=b M / a$ particles located in the strip at the right edge of the cell. Using the collision rules (2) and (3), one finds

$$
\left\langle\left(\sum_{i=1}^{P} \Delta v_{i}^{2}\right)^{2}\right\rangle=16\left(k_{B} T\right)^{2}(1-c) P(M-P) / M^{2} .
$$

Inserting $P=b M / a$ and integrating over $b$, we have, finally,

$$
\left\langle\sigma_{T, r o t}^{2}(0)\right\rangle=\frac{8}{3 M} a^{2} N(1-c)\left(k_{B} T\right)^{2},
$$

so that the rotational contribution to the thermal diffusivity at time zero is

$$
D_{T, r o t}(0)=\frac{1}{M} \frac{a^{2}}{6 \tau}(1-c) .
$$

It is now clear why collisions do not make a significant contribution to the thermal diffusivity, even at small $\lambda / a$. The correlations of the heat flux contain the internal energy, which is proportional to the sum of the squares of the relative velocity vectors. The special nature of the collision step in stochastic rotation dynamics (SRD) — rotations of the relative velocity vectors-leaves the length of these vectors invariant. For large $M$, the relative velocity of a particle is essentially equal to the velocity itself, so that rotations do not transfer heat. This is the source of the factor $1 / M$ in $D_{T, \text { rot }}$, implying that collision contributions to the thermal conductivity are negligible in practical applications, where $M$ is generally much larger than 1. Evaluating Eq. (95) for the parameters of Fig. 14, $M=5, \tau=1, k_{B} T=0.0413, c=0.5$, $a=1$ gives $D_{T, \text { rot }}(0)=0.01666$, while the measured value from Fig. 14 is 0.012275 , which differs only by a factor of 1.357. This difference might be due to the fact that we have neglected fluctuations in the number of particles in a cell; for $M=5$, these fluctuations are not negligible.

\section{ANALYSIS OF THE SELF-DIFFUSION CONSTANT}

The self-diffusion constant $D$ of particle $i$ is defined by

$$
D=\lim _{t \rightarrow \infty} \frac{1}{2 d t}\left\langle\left[\mathbf{r}_{i}(t)-\mathbf{r}_{i}(0)\right]^{2}\right\rangle
$$

The position of the particle at time $t=n \tau$ is

$$
\mathbf{r}_{i}(t)=\mathbf{r}_{i}(0)+\tau \sum_{i=0}^{n-1} \mathbf{v}_{i}(k \tau)
$$

so that

$$
\left\langle\left[\mathbf{r}_{i}(t)-\mathbf{r}_{i}(0)\right]^{2}\right\rangle=\tau^{2} \sum_{j=0}^{n-1} \sum_{k=0}^{n-1}\left\langle\mathbf{v}_{i}(j \tau) \cdot \mathbf{v}_{i}(k \tau)\right\rangle .
$$

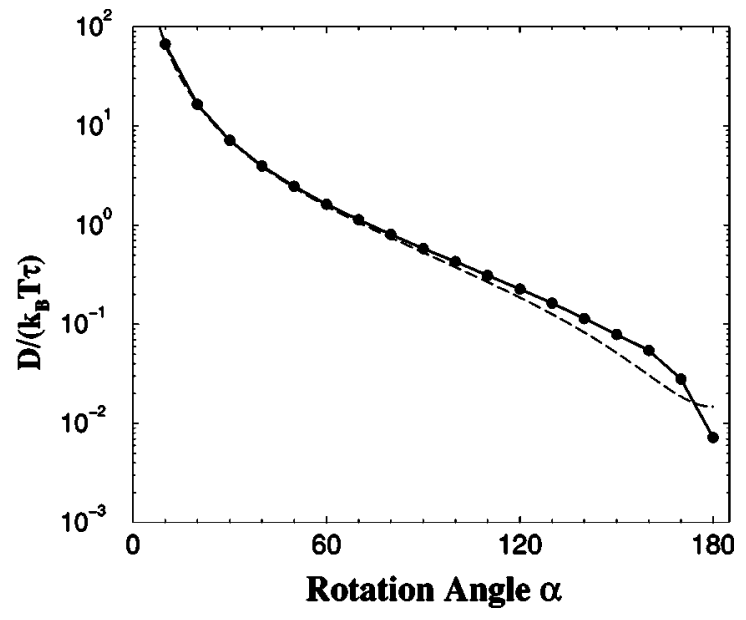

FIG. 15. Dimensionless self-diffusion constant $D /\left(k_{B} T \tau\right)$ as a function of the rotation angle $\alpha$ (solid line and bullets). The dashed line is a plot of approximation (101). Parameters: $M=35, k_{B} T$ $=0.01275, \lambda / a=0.113, L=32$.

The sums can be rewritten as

$$
\begin{aligned}
& \sum_{j=0}^{n-1} \sum_{k=0}^{n-1}\left\langle\mathbf{v}_{i}(j \tau) \cdot \mathbf{v}_{i}(k \tau)\right\rangle \\
& \quad=\sum_{j=0}^{n-1}\left\langle v_{i}^{2}(j \tau)\right\rangle+2 \sum_{j=0}^{n-2} \sum_{k=j+1}^{n-1}\left\langle\mathbf{v}_{i}(j \tau) \cdot \mathbf{v}_{i}(k \tau)\right\rangle \\
& =n d k_{B} T+2 \sum_{j=1}^{n-1} j\left\langle\mathbf{v}_{i}(0) \cdot \mathbf{v}_{i}(n-j) \tau\right\rangle .
\end{aligned}
$$

Restricting ourselves to $d=2$, and making the same approximations as in Sec. II A, one has

$$
\left\langle\mathbf{v}_{i}(0) \cdot \mathbf{v}_{i}(k \tau)\right\rangle=2 \zeta_{1}^{k} k_{B} T
$$

so that

$$
D=\lim _{n \rightarrow \infty} \frac{\tau^{2}}{4 n \tau}\left[2 n k_{B} T+2 \sum_{j=1}^{n-1} j \zeta_{1}^{n-j}\right]=k_{B} T \tau\left[\frac{1}{2}+\frac{\zeta_{1}}{1-\zeta_{1}}\right]
$$

The same result can be obtained using the discrete GreenKubo relation

$$
D=\frac{\tau}{d} \sum_{t=0}^{\infty}\left\langle\mathbf{v}_{i}(0) \cdot \mathbf{v}_{i}(t)\right\rangle
$$

In Fig. 15, the diffusion constant (measured as small times) is plotted as a function of the rotation angle and compared with Eq. (101). For angles not too close to $180^{\circ}$, the agreement is very good. It follows from Eqs. (101), (51), and (71) that the Schmidt number $\mathrm{Sc}=\nu / D$ is smaller than or of the order of 1 for large mean free path. However, for $\lambda / a$ $\ll 1$, very large Schmidt numbers can be obtained since Sc $\sim(a / \lambda)^{2}$. Sc can be further increased by going to larger rotation angles, where $D$ becomes very small. 


\section{Correction term}

For $k \geqslant 2$, Eq. (100) is not exact. It neglects contributions from situations in which particles that are in the same box at time $k_{1} \tau, 0 \leqslant k_{1}<k$, are again in the same box at a later time $k_{2} \tau, k_{1}<k_{2} \leqslant k$. In contrast to the situation with the viscosity, these terms are not of the order of $1 / M^{2}$ or smaller; they are of the order of $\sim(1-c) / M$, i.e., of the same order as the leading corrections for finite particle number $M$. The probability that such multiple encounters occur increases the longer a particle stays in the same cell, which is the case for small mean free path and strong backscattering, i.e., at large angles $\alpha$. This is consistent with the results shown in Fig. 15 for $\lambda / a=0.11$, where deviations at larger angles can be seen. A detailed analysis of these corrections goes beyond the scope of this paper; however, we can gain some insight into these corrections if we consider the worst case scenario of zero mean free path. In this case, particles remain in the same cell, and

$$
\left\langle\mathbf{v}_{i}(0) \cdot \mathbf{v}_{i}(k \tau)\right\rangle=2 k_{B} T\left(c^{k}+\frac{1-c^{k}}{M}\right) .
$$

As expected, this result differs from Eq. (100) by terms of $O(1 / M)$ for $k \geqslant 2$. Although this $\lambda / a \rightarrow 0$ limiting case is pathological, it does show the existence of additional $O(1 / M)$ corrections to the self-diffusion constant.

\section{LONG-TIME TAILS}

There is a renormalization of the transport coefficientsthe viscosity, the diffusion constant, and the thermal diffusivity - at long times due to long-time tails in the corresponding autocorrelation functions [6,7]. In two dimensions, these correlation functions are predicted to behave as $1 / t$ at large times, and this leads to transport coefficients that diverge logarithmically with time. Specifically, mode-coupling theory predicts

$$
\left\langle\sigma_{x y}(t) \sigma_{x y}(0)\right\rangle \sim \frac{\left(k_{B} T\right)^{2}}{\rho d(d+2)}\left(\frac{d^{2}-2}{(8 \pi \nu t)^{d / 2}}+\frac{1}{\left(4 \pi \Gamma_{s} t\right)^{d / 2}}\right)
$$

and

$$
\left\langle v_{x}(t) v_{x}(0)\right\rangle \sim \frac{d-1}{\rho d} \frac{k_{B} T}{[4 \pi(\nu+D) t]^{d / 2}}
$$

at large times, where $D$ is the bare self-diffusion constant, $\rho$ is the number density ( $\sim M$ in our method), $d$ is the dimension, and

$$
\Gamma_{s}=\frac{2(d-1)}{d} \nu+\gamma+\left(\frac{c_{p}}{c_{v}}-1\right) D_{T}
$$

is the sound attenuation coefficient, where $D_{T}=\lambda_{T} c_{v} / c_{p}$ is the thermal diffusivity, and the thermal transport coefficient $\lambda_{T}$ is defined in Eq. (57) of Part 1.

In order to resolve the tails in a simulation, parameters have to be optimized to obtain a large signal to noise ratio.

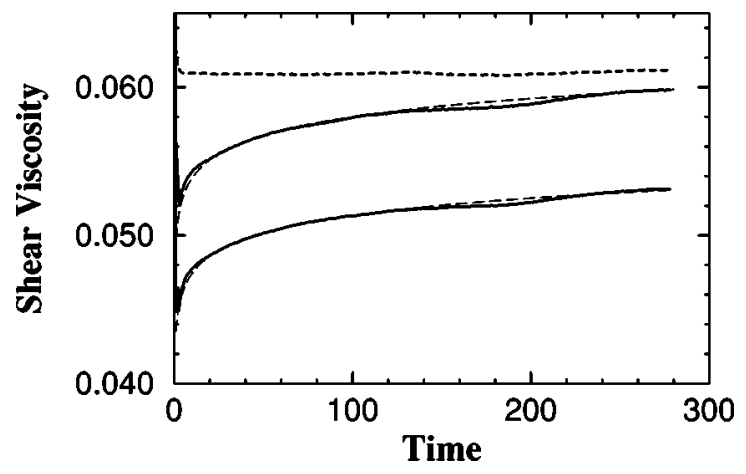

FIG. 16. Various contributions to the shear viscosity as a function of time. The lower solid line is the kinetic part $\nu_{k i n}$ and the upper solid line is total viscosity $\nu$. Both exhibit the same logarithmic behavior. The function $a+b \ln (t)$ (long dashed lines) is shown for comparison. The deviation at time $t \approx 200$ is caused by the recurrence of sound waves. The top dashed line is the rotational contribution $\nu_{\text {rot }}$ which is essentially constant at long times. Parameters: $\alpha=60^{\circ}, \lambda / a=0.2, M=5, k_{B} T=0.0413$. Lower curve, $a$ $=0.0435, b=0.0017$; upper curve, $a=0.0497, b=0.0018$. Time average over 130000 time steps and over 40 different initial seeds of the random number generator.

First, from Eqs. (104) and (105) it can be seen that the amplitudes of the tails are large at low density and small viscosity. Hence, we take a small number of particles per cell, $M=5$ and $M=10$. Furthermore, in order to have a small viscosity and diffusion constant, the temperature should not be too large. High temperature also means a large speed of sound, $c_{s}=\sqrt{2 k_{B} T}$, so that the time $t_{r}=L / c_{s}$ for the recurrence of sound waves would be too small. On the other hand, the physical origin of the long-time tails is the so-called backflow effect, in which a moving particle creates vortices that couple back to it and push it further in the already chosen direction. These vortices need some time $t_{S}$ to develop. A final requirement is that there be no other slow relaxation process which could mask the tail. This means that tails can only be observed in the time window between $t_{S}$ and $t_{r}$; furthermore, the smaller the value of $t_{S}$, the larger the signal to noise ratio. We found in the simulations that $t_{S}$ also decreases with increasing temperature.

Although the viscosity in $d=2$ has a minimum at $\alpha$ $=90^{\circ}$ for moderate to large mean free paths, as discussed above, the oscillatory decay of the kinetic stress correlations in this case is very slow. In order to avoid the possibility that this slow decay could interfere with the measurement of the long-time tails, a rotation angle $\alpha=60^{\circ}$ was chosen, for which the decay is rather fast. In order to reduce the statistical noise to acceptable levels, a time average of the stress correlations was taken over 130000 time steps, and an average over the $x$ and $y$ directions was performed. We also averaged over 40 runs with different initial conditions. For this set of parameters and $L=64$, we were able to directly measure the logarithmic behavior of the shear viscosity; see Fig. 16. We found that only the kinetic part of the stress tensor contributes to the $1 / t$ tail in the stress correlation function; no tails could be detected in either the rotational or mixed contributions. 
If we use Eq. (104) in the continuum Green-Kubo relation for the shear viscosity, the asymptotic behavior of the viscosity in two dimensions is predicted to be

$$
\nu(t) \sim k_{B} T d_{0} \ln (t),
$$

with $d_{0}=a^{2}\left(1 / \nu+1 / \Gamma_{s}\right) /(32 \pi M)$, where $a$ is the cell dimension. At time $t=100$ we measured a shear viscosity $\nu$ $=0.058$ and a thermal diffusivity $D_{T}=0.096$. The bulk viscosity was zero within the error bars. Using the $d=2$ ideal gas value $c_{p} / c_{v}=2$, we obtain the theoretical prediction $d_{0}$ $=0.0475$. A logarithmic fit of the measured temporal behavior of the viscosity gives

$$
\nu \sim 0.0492+0.00188 \ln (t),
$$

leading to $d_{0}=0.04552$, which is only $4.5 \%$ smaller than the value predicted by theory. In order to investigate the density dependence of the long-time tails, another simulation was conducted with twice as many particles per cell, $M=10$. We found that the amplitude $d_{0}$ of the tail was exactly half that measured for $M=5$, as expected.

We have also measured the amplitude of the long-time tail of the velocity autocorrelation function, and obtained a result that is within $15 \%$ of the predicted value. The numerical effort in this case was smaller than for the viscosity measurement because we could also average over all particles. In the simulations of $\left\langle v_{i x}(0) v_{i x}(t)\right\rangle$ we also performed an ensemble average over 1000-2000 different initializations of the random number generator, which required CPU times between 5 and $10 \mathrm{~h}$ for $L=64$ and $M=5-10$. Figure 17(a) shows the $1 / t$ tail of the velocity autocorrelation function for two different particle densities, $M=5$ and $M=10$. Again, we observe that the amplitude is proportional to $\sim 1 / M$, as predicted by Eq. (105). Figure 17(b) contains plots of the normalized velocity autocorrelation function $\left\langle v_{i x}(0) v_{i x}(t)\right\rangle t_{S} /\left[\tau\left\langle v_{i x}(0)^{2}\right\rangle\right]$ as a function of the scaled time $t / t_{S}$, where $t_{S}=L / \sqrt{2 k_{B} T}$ is the recurrence time for sound waves, for two temperatures, $k_{B} T=0.01275$ and 0.0413 . Both curves show the same behavior, namely, a peak at large times, $t / t_{S} \sim L$, due to the recurrence of sound waves, as well as the onset of the tail at the same rescaled time.

As an additional test for this scaling behavior, we coupled to a heat bath using a stochastic method similar to that described in Ref. [11], and found that the correlation functions in the canonical ensemble are the same as in the microcanonical ensemble if the time is rescaled by a factor of $\sqrt{2}$. This is exactly the ratio of the adiabatic and isothermal speeds of sound, i.e., the thermalization reduces the speed of sound from $\sqrt{2 k_{B} T}$ to $\sqrt{k_{B} T}$. Both $t_{r}$ and $t_{S}$ are therefore determined by the speed of sound.

We also measured the long-time behavior of the thermal diffusivity, Fig. 14, and the bulk viscosity, Fig. 12. While we can see a logarithmic tail in $D_{T}$, the bulk viscosity remains essentially zero at all times.

In summary, the SRD algorithm has passed a very sensitive test. The amplitude of the long-time tail of the stress
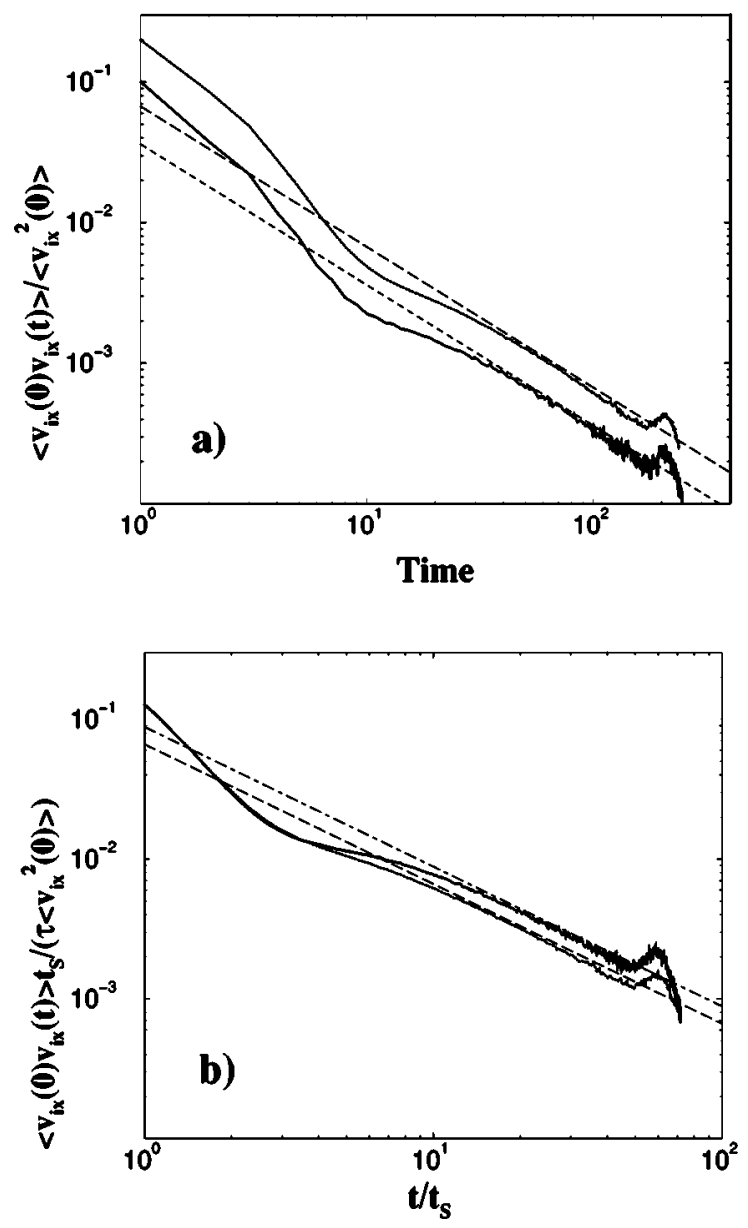

FIG. 17. Long-time tail of the normalized velocity autocorrelation function averaged over all particles and 2000 initial seeds of the random number generator. (a) $\left\langle v_{i x}(0) v_{i x}(t)\right\rangle /\left\langle v_{i x}^{2}(0)\right\rangle$ vs time for $M=5$ (upper dotted curve) and $M=10$ (solid curve). The longdashed line is the fit $0.067 / t$. The dashed line is the fit $0.036 / t$. Parameters: $\alpha=90^{\circ}, k_{B} T=0.0413, L=64, \tau=1$. (b) The normalized velocity autocorrelation function $\left\langle v_{i x}(0) v_{i x}(t)\right\rangle t_{S} /\left[\tau\left\langle v_{i x}^{2}(0)\right\rangle\right]$ as a function of the scaled time $t / t_{S}$, where $t_{S}=L / \sqrt{2 k_{B} T}$ is the recurrence time for sound waves. The solid curve corresponds to $k_{B} T=0.01275$, while the dotted curve is for a larger temperature $k_{B} T=0.0413$. The dash-dotted line is the fit $0.0884 / t$, while the dashed line is the fit $0.0663 / t$. Other parameters: $\alpha=90^{\circ}, M=5$, $L=64, \tau=1$.

correlation function is a sensitive function of the nonlinearities in the hydrodynamic equations and the possible existence of spurious invariants, such as occur in conventional lattice gas automata (LGA) [8,9]. Simulations of LGA showed a long-time tail in the stress correlations, but the prefactor is altered by spurious invariants. To our knowledge, molecular dynamics simulations with soft potentials have, so far, failed to confirm this tail [10], probably because the kinetic contribution to the stress tensor, which is responsible for the tail, is much smaller than the potential contribution. With SRD it is possible to tune the relative amplitude of the relative kinetic and potential (rotational) contributions, making it possible to measure the individual contributions directly. 


\section{CONCLUSION}

In this paper, we have presented a comprehensive analytical and numerical study of the stochastic rotation dynamics model for fluid dynamics in two dimensions. Exact calculations-incorporating both the cell structure and the random cell shifts prior to collisions-were presented, and it was shown that finite cell size effects persist even at large mean free path. These corrections to the continuum approximation (which amounts to ignoring contributions from $\Delta \boldsymbol{\xi}^{s}$ and replacing $\Delta \boldsymbol{\xi}$ by $\tau \mathbf{v}$ in stress correlation functions) were shown to lead to an additional contribution to the shear viscosity which is significant for $\alpha \approx 90^{\circ}$ in two dimensions. The resulting expression for the viscosity was shown to be in excellent agreement with simulations, thus resolving discrepancies with previous theoretical results. Accurate explicit expressions for all other transport coefficients were derived and compared with simulations for a wide range of mean free paths and rotations angles. Several approximations and assumptions were discussed in detail and their validity tested. No assumptions were made regarding the validity of the mo- lecular chaos approximation, so that correlation effects, which are particularly important for small values of the ratio $\lambda / a$, were incorporated into the calculations. This extends the original work of Malevanets and Kapral [2] to arbitrary rotation angles and small temperatures, and allowed us to obtain accurate approximations for the transport coefficients. Finally, as a very sensitive test of the model, long-time tails in the velocity, stress, and heat-flux autocorrelation functions were analyzed and shown to be in excellent agreement with the results of previous mode-coupling calculations.

\section{ACKNOWLEDGMENTS}

Support from the National Science Foundation under Grant Nos. DMR-9712134 and DMR-0083219, and the donors of The Petroleum Research Fund, administered by the ACS, are gratefully acknowledged. T.I. thanks G. Gompper for his hospitality at the IFF, Forschungszentrum Jülich, and J.R. Dorfman for answering questions about long-time tails. We also thank E. Tüzel, M. Strauss, and F. Tzschichholz for valuable discussions.
[1] A. Malevanets and R. Kapral, J. Chem. Phys. 110, 8605 (1999).

[2] A. Malevanets and R. Kapral, J. Chem. Phys. 112, 7260 (2000).

[3] T. Ihle and D.M. Kroll, Phys. Rev. E 63, 020201(R) (2001).

[4] P.M. Morse and H. Feshbach, Methods of Theoretical Physics (McGraw-Hill, New York, 1953).

[5] T. Ihle and D.M. Kroll (unpublished).

[6] M.H. Ernst, E.H. Hauge, and J.M.J. van Leeuwen, Phys. Rev. Lett. 25, 1254 (1970).
[7] M.H. Ernst, E.H. Hauge, and J.M.J. van Leeuwen, Phys. Rev. A 4, 2055 (1971).

[8] L.P. Kadanoff, G.R. McNamara, and G. Zanetti, Phys. Rev. A 40, 4527 (1989).

[9] T. Naitoh, M.H. Ernst, and J.W. Dufty, Phys. Rev. A 42, 7187 (1990).

[10] M. Ferrario, A. Fiorino, and G. Cicotti, Physica A 240, 268 (1997).

[11] D.M. Heyes, Chem. Phys. 82, 285 (1983). 